\title{
Film Dynamics and Lubricant Depletion by Droplets Moving on Lubricated Surfaces
}

\author{
Michael J. Kreder, ${ }^{1}$ Dan Daniel, ${ }^{1,2}$ Adam Tetreault, ${ }^{3}$ Zhenle Cao, ${ }^{3}$ Baptiste Lemaire, ${ }^{1}$ \\ Jaakko V. I. Timonen, ${ }^{1,5}$ and Joanna Aizenberg ${ }^{1,3,4, *}$ \\ ${ }^{1}$ John A. Paulson School of Engineering and Applied Sciences, Harvard University, \\ Cambridge, Massachusetts 02138, USA \\ ${ }^{2}$ Institute for Materials Research and Engineering, 2 Fusionopolis Way, Singapore 138634 \\ ${ }^{3}$ Wyss Institute for Biologically Inspired Engineering, Harvard University, \\ Cambridge, Massachusetts 02138, USA \\ ${ }^{4}$ Department of Chemistry and Chemical Biology, Harvard University, \\ Cambridge, Massachusetts 02138, USA \\ ${ }^{5}$ Department of Applied Physics, Aalto University School of Science, Espoo FI-02150, Finland
}

(Received 25 January 2018; revised manuscript received 11 June 2018; published 4 September 2018)

\begin{abstract}
Lubricated surfaces have shown promise in numerous applications where impinging foreign droplets must be removed easily; however, before they can be widely adopted, the problem of lubricant depletion, which eventually leads to decreased performance, must be solved. Despite recent progress, a quantitative mechanistic explanation for lubricant depletion is still lacking. Here, we first explain the shape of a droplet on a lubricated surface by balancing the Laplace pressures across interfaces. We then show that the lubricant film thicknesses beneath, behind, and wrapping around a moving droplet change dynamically with the droplet's speed-analogous to the classical Landau-Levich-Derjaguin problem. The interconnected lubricant dynamics results in the growth of the wetting ridge around the droplet, which is the dominant source of lubricant depletion. We then develop an analytic expression for the maximum amount of lubricant that can be depleted by a single droplet. Counterintuitively, faster-moving droplets subjected to higher driving forces deplete less lubricant than their slower-moving counterparts. The insights developed in this work will inform future work and the design of longer-lasting lubricated surfaces.
\end{abstract}

DOI: 10.1103/PhysRevX.8.031053

\section{INTRODUCTION}

The ability of liquid lubricant on surfaces to reduce solid-solid friction has been widely known since antiquity [1,2]; examples include the ubiquitous use of lubricant oil between the moving parts of a machine and the synovial fluid found naturally in the joint cavities of our bodies [3,4]. The idea of using lubricant to reduce solid-liquid friction is relatively new: When infused with suitable lubricants, surfaces can exhibit excellent liquid repellency [5-7]. Such surfaces known in the literature as slippery lubricantinfused porous surfaces (SLIPS) also show promise in various applications, including biomedical devices and anti-ice materials [8-12]. The origin of the repellency in SLIPS is largely due to the presence of a stable lubricant film above the solid surface; however, lubricant can be lost

*jaiz@seas.harvard.edu

Published by the American Physical Society under the terms of the Creative Commons Attribution 4.0 International license. Further distribution of this work must maintain attribution to the author(s) and the published article's title, journal citation, and DOI.
Subject Areas: Fluid Dynamics, Materials Science, Soft Matter due to various factors (body forces, evaporation or solubility, shear, etc.), eventually leading to decreased performance $[13,14]$. Many strategies have been proposed to retain the lubricant overlayer, ranging from the choice of structures (nanoscale vs microscale, periodic vs random, etc.) $[15,16]$ to the choice of lubricant (high- vs lowviscosity lubricant), and finally to the use of patterned wettability on a surface [17].

Despite recent progress, a quantitative mechanistic understanding of lubricant depletion due to a moving droplet has not been reported in the literature. Here, we begin by using geometric arguments and quasistatic approximations - when balancing Laplace pressures across various interfaces - to deduce the shape of a droplet on a lubricated surface. We then proceed to establish scaling arguments for the dynamic behavior of lubricant around a moving droplet by greatly expanding on the LandauLevich-Derjaguin (LLD) analysis first presented by Daniel et al. [18] for the description of the film thickness under the moving droplet. We validate this model by using whitelight interferometry to measure the dynamically changing lubricant thicknesses behind, underneath, and wrapping around a moving water droplet. 
In our previous work, we showed that the LLD analysis can be used to model droplet mobility on lubricated surfaces [18]. Here, we extend this analysis to directly model lubricant depletion and demonstrate the important role of the wetting ridge by showing explicitly that its growth is the dominant source of depletion.

\section{RESULTS AND DISCUSSION}

\section{A. Droplet geometry and Laplace pressures on SLIPS}

Recent work by Semprebon et al. [19] and Tress et al. [20] used numerical methods to solve the Young-Laplace equation for the droplet geometry on a lubricant-infused surface. Our analysis is consistent with previous work, but we make a number of simplifying assumptions-for the case when the wetting ridge is much smaller than the droplet - that allow for an analytical solution and a simple physical interpretation for the geometry of a millimetric sized droplet on well-designed SLIPS.

As shown in Fig. 1(a), there are three important length scales to consider: the external radius of the wetting ridge $r_{\text {ext }}$, the internal radius of the wetting ridge $r_{\text {int }}$, and the radius of the droplet itself $R$, whereby $r_{\text {ext }} \sim r_{\text {int }} \ll R$. Note that the micron thicknesses of the lubricant on the substrate outside the droplet, underneath the droplet, and wrapping around the droplet are much thinner than the size of the wetting ridge and do not directly affect the droplet geometry. In our schematic, there is a stable lubricant film underneath the droplet, meaning that there is no welldefined contact angle between the lubricant and the solid [18]. While this is not always the case, the contact angle that the lubricant makes with the solid substrate is close to zero for many well-designed surfaces, even in the absence of a stable intercalating film [21].

We begin by considering a droplet with a wrapping layer of lubricant over it, which occurs when the spreading coefficient of lubricant over the droplet is positive, that is, $S_{\mathrm{LD}}=\gamma_{\mathrm{DV}}-\gamma_{\mathrm{LV}}-\gamma_{\mathrm{LD}}>0$, where $\gamma_{\mathrm{DV}}, \gamma_{\mathrm{LV}}$, and $\gamma_{\mathrm{LD}}$ are the interfacial energies of the droplet-vapor, lubricantvapor, and lubricant-droplet interfaces, respectively [21,22].
The geometry of the sessile droplet, ignoring the effects of gravity, can be understood by equating the Laplace pressures across the different interfaces in the system. The pressure in the drop $P_{\text {drop }}$ can be deduced by applying the YoungLaplace equation across the two interfaces of the wrapping layer, giving

$$
P_{\text {drop }}=P_{\mathrm{atm}}+\frac{2 \gamma_{\mathrm{eff}}}{R}=P_{\mathrm{atm}}+\frac{2\left(\gamma_{\mathrm{LV}}+\gamma_{\mathrm{LD}}\right)}{R},
$$

while the pressure in the wetting ridge can be deduced from the Laplace pressure either across the air-lubricant or lubricant-droplet interface, giving

$$
\begin{aligned}
P_{\text {ridge }} & =P_{\mathrm{atm}}-\gamma_{\mathrm{LV}}\left(\frac{1}{r_{\mathrm{ext}}}-\frac{1}{a}\right) \\
& =P_{\mathrm{drop}}-\gamma_{\mathrm{LD}}\left(\frac{1}{r_{\mathrm{int}}}+\frac{1}{a}\right),
\end{aligned}
$$

where $a$ is the base radius of the droplet.

Comparing Eqs. (1) and (2) and noting that $R \approx a$ for droplets with $\theta_{\text {app }} \approx 90^{\circ}$, which is true for water droplets on typical SLIPS, we find that

$$
\frac{\gamma_{\mathrm{LD}}}{r_{\mathrm{int}}}=\frac{\gamma_{\mathrm{LV}}}{r_{\mathrm{ext}}}+\frac{\gamma_{\mathrm{LV}}+\gamma_{\mathrm{LD}}}{R},
$$

where the droplet radius $R$ is set by the volume of the droplet $V$ and the apparent contact angle $\theta_{\text {app }}$ it makes with the surface, i.e., $V=(\pi / 3) R^{3}\left(2+\cos \theta_{\text {app }}\right)\left(1-\cos \theta_{\text {app }}\right)^{2}$.

To verify Eq. (3), we image the wetting ridge using fluorescence confocal microscopy [Fig. 1(b)] [21]. We measure $R, r_{\text {int }}$, and $r_{\text {ext }}$ for droplets of 3 and $8 \mu \mathrm{l}$ and find good agreement (within $3 \%$ ) between values predicted from Eq. (3) and experimental values (see Appendix A, Tables I and II).

There has been some debate over the correct physical interpretation of $\theta_{\text {app }}$ for SLIPS, which is the angle observed using conventional optical contact-angle instruments $[21,23]$. Interestingly, a lubricated surface approaches an (a)

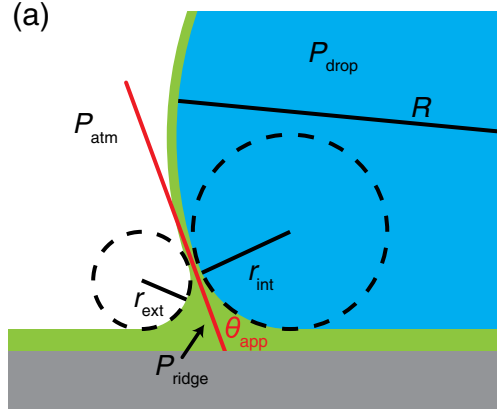

(b)

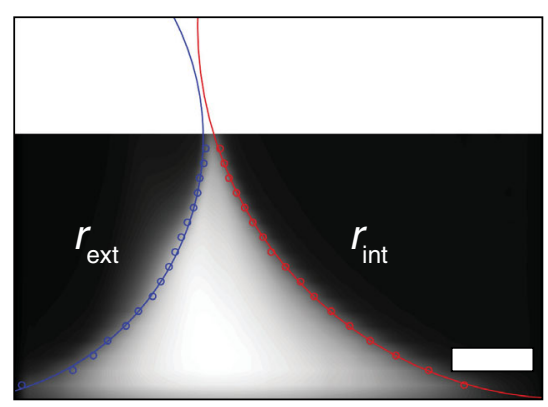

(c)

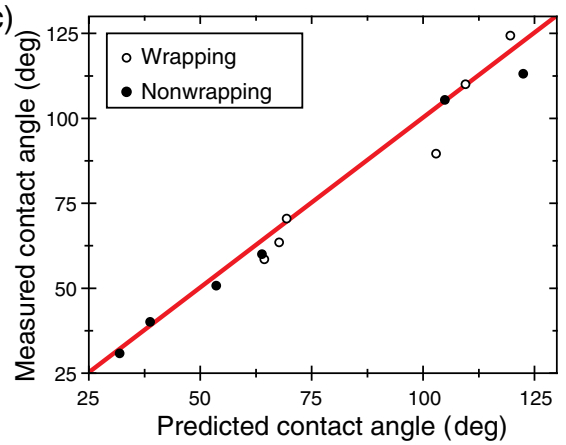

FIG. 1. (a) Schematic showing the geometry of a droplet when a wrapping layer is present $\left(S_{\mathrm{LD}}>0\right)$. (b) Confocal image confirming the profile of the wetting ridge (scale bar $=25 \mu \mathrm{m}$ ) around a droplet on fluorescently dyed silicone oil. (c) Measured apparent contact angle vs predicted apparent contact angle based on Eq. (4). The red line indicates a slope of 1. 
(a)

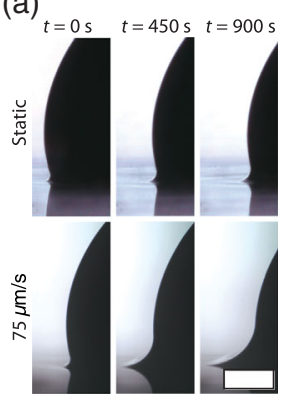

(b)

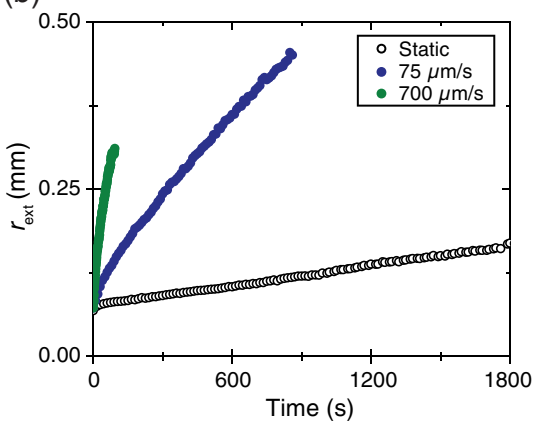

FIG. 2. (a) Time-lapse images of the wetting ridge for static and moving droplets (scale bar $=0.5 \mathrm{~mm}$ ). (b) Growth of $r_{\mathrm{ext}}$ over time for static and moving droplets. As can be seen in both (a) and (b), the wetting ridge grows more quickly for the moving droplets. In all cases, $25-\mu 1$ droplets are placed on a surface infused with $20-\mathrm{cP}$ silicone oil with an initial film thickness of $4 \mu \mathrm{m}$.

idealized Young's surface for a vanishingly small wetting ridge since there is no contact line pinning for an atomically smooth liquid-liquid interface. Hence, $\theta_{\text {app }}$ can be described by a modified Young's equation

$$
\cos \theta_{\text {app }}=\frac{\gamma_{\mathrm{LV}}-\gamma_{\mathrm{LD}}}{\gamma_{\mathrm{eff}}}
$$

where the solid phase is replaced by the lubricant oil (l) phase, and $\gamma_{\mathrm{eff}}=\gamma_{\mathrm{LV}}+\gamma_{\mathrm{LD}}$ or $\gamma_{\mathrm{DV}}$ for droplets with and without a wrapping layer, respectively [19]. Equation (4) can be obtained by either minimizing energy or by balancing forces due to the interfacial tensions at the ridge, similar to the argument originally proposed by Young [24] (Appendix B, Fig. 7). Alternatively, $\theta_{\text {app }}$ can be deduced using purely geometrical considerations (Fig. 8). As shown in Fig. 1(c), there is good agreement between experimentally measured contact angles - both by this group [6] and others [21] - and the contact angles predicted by Eq. (4) [see Appendix B, Table III for data used in Fig. 1(c)].

Equations (3) and (4) are true only when $r_{\mathrm{ext}} \ll R$ and $r_{\mathrm{ext}} \ll l_{c}$, where $l_{c}=\left(\gamma_{\mathrm{LV}} / \rho_{l} g\right)^{1 / 2} \sim \mathrm{mm}$ is the capillary length for the lubricant of density $\rho_{l}$.

The wetting ridge is a low-pressure region, and it will grow in size until $r_{\mathrm{ext}} \sim R$ or $r_{\mathrm{ext}} \sim l_{c}$ as lubricant flows from the surrounding area into the ridge, analogous to the flow of liquid from the lamellae into the plateau borders in liquid foams [25]. Equations (3) and (4) no longer hold as the wetting ridge size grows and approaches $l_{c}$. In this case, it can no longer be approximated as the arc of a circle with radius $r_{\mathrm{ext}}$, but it is described instead by a Bessel function $[21,26]$. In practice, for millimetric droplets on micronthick lubricant films, the growth of $r_{\mathrm{ext}}$ around a static droplet is limited by thin-film flow and does not approach $l_{c}$ even after a long time. For example, a 25- $\mu$ l droplet sitting on $4-\mu$ m-thick 20 -cP silicone oil has a wetting ridge with an initial $r_{\mathrm{ext}} \approx 50 \mu \mathrm{m}$, which grows only to about $150 \mu \mathrm{m}$ after $30 \mathrm{~min}$ (Fig. 2). The wetting ridge can, however, grow considerably faster for a moving droplet, as we explore more fully in the following section.

\section{B. Lubricant dynamics}

To understand the lubricant dynamics entrained by moving droplets, we track the size of the wetting ridge and the thickness of the lubricant in key position with time [Fig. 3(a)]. The droplet is held in place by a capillary tube, while the SLIPS sample with initial film thickness $h_{i}$ is moved at controlled speeds $U=75-700 \mu \mathrm{m} / \mathrm{s}$ using a linear motor. We find that pinning the droplet above a moving surface has a negligible effect on the droplet behavior compared to the more conventional case of a droplet moving on an inclined surface (Appendix C, Fig. 9). In all of our experiments, the SLIPS samples consist of randomly oriented nanoplates of size $10 \mathrm{~nm}$, spaced $200 \mathrm{~nm}$ apart on glass substrates [15].

The spatial distribution of the lubricant around and under a moving droplet can be observed using reflection interference contrast microscopy (RICM) [Fig. 3(b)] [18,28]. Briefly, we shine a monochromatic light of wavelength

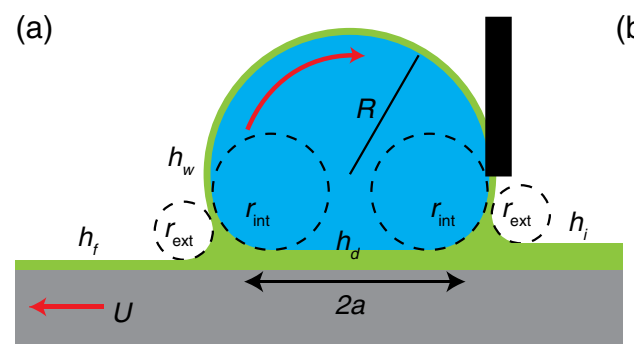

(b)

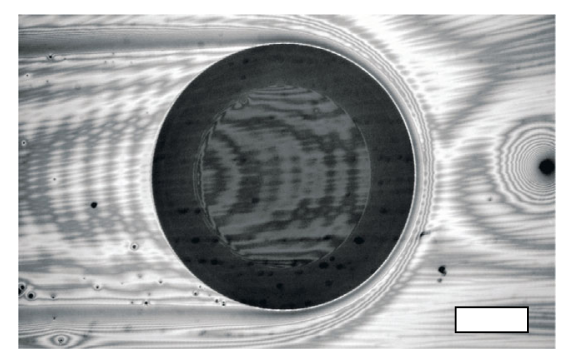

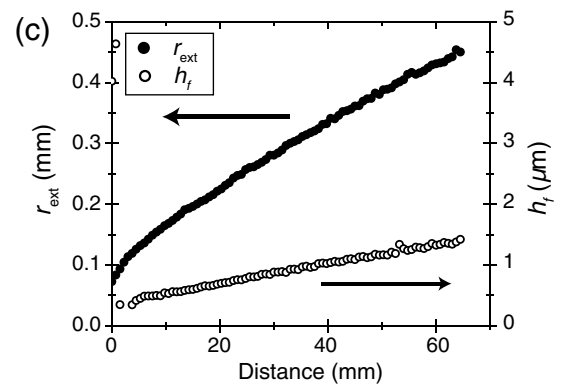

FIG. 3. (a) Schematic of the experimental setup used to study the lubricant dynamics. The substrate is moved while the droplet is held in place by a capillary tube, allowing for measurement of the wetting ridge and lubricant thicknesses in various positions. (b) RICM image demonstrating the lubricant profile around a moving droplet (scale bar $=1 \mathrm{~mm}$ ). See the Supplemental Material Video S1 [27] for lubricant dynamics visualized using RICM. (c) Typical experimental measurement of $h_{f}$ and $r_{\text {ext }}$ for a droplet moving on a lubricantinfused surface. The data for the first two open circles refer to initial thickness $h_{i} \approx 4 \mu \mathrm{m}$. 
$\lambda=532 \mathrm{~nm}$ from beneath a transparent substrate, and in the presence of a thin lubricant film, light reflected off the two film interfaces interferes either constructively or destructively to form bright or dark fringes, respectively. Between two bright or dark fringes, there is a difference in film thickness of $\lambda / 2 n_{\text {lub }}$, where $n_{\text {lub }}$ is the refractive index of the lubricant film. The uniformly dark region around the droplet corresponds to the wetting ridge as light that reflects off the angled ridge is not collected by the objective (Appendix D).

The external radius of the wetting ridge $r_{\text {ext }}$ either at the advancing or receding front is also monitored optically from the side using a high-resolution camera fitted with a microscope objective or a telecentric lens. At the same time, the thickness of the initial lubricant film $h_{i}$, behind the moving droplet $h_{f}$, under the droplet $h_{d}$, and wrapping around the droplet $h_{w}$ are measured using white-light interferometry. White light reflecting off the thin film is collected by an optical fiber of spot size approximately $50 \mu \mathrm{m}$ and analyzed using a spectrometer. Thicknesses in the range between hundreds of nanometers to tens of microns can be determined this way; details of the setup have been reported in our previous work [18]. In our experiment, $h_{f}, h_{d}$, and $h_{i}$ are measured along the midline of the droplet profile, where the lubricant profile is nearly flat with $\Delta h$ of at most $\lambda / 4 n_{\text {lub }} \sim 100 \mathrm{~nm}$.

Experimentally, we find that both $r_{\mathrm{ext}}$ and $h_{f}$ grow (initially) with the distance traveled by the droplet [Fig. 3(c)] as the lubricant is depleted. We can understand the scaling of $h_{f}$ and $r_{\text {ext }}$, since this behavior is analogous to the classical LLD problem [18,29-31]. There is a pressure difference between the wetting ridge and the trailing lubricant film behind the droplet that must be balanced by viscous dissipation in the transition region of size $d_{f}$ [bounded in red in Fig. 4(a)]. The film thickness $h_{f}$ can be deduced by balancing $\nabla P$ and $\eta \nabla^{2} U$ in this region, i.e., $\left(\gamma_{\mathrm{LV}} / d_{f}\right)\left(1 / r_{\mathrm{ext}}-1 / R\right) \sim \eta U / h_{f}^{2}$ and matching the curvature in this transition region $\partial^{2} h_{f} / \partial^{2} x \sim h_{f} / d_{f}^{2}$ with that of the wetting ridge $1 / r_{\text {ext }}$, i.e., $d_{f} \sim \sqrt{r_{\text {ext }} h_{f}}$. Combining these relations gives us the following scaling:

$$
h_{f} / r_{\mathrm{ext}}\left(1-r_{\mathrm{ext}} / R\right)^{2 / 3} \sim C a_{\mathrm{LV}}^{2 / 3}
$$

(a)

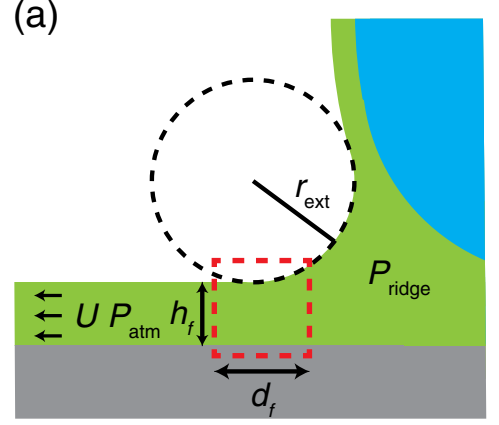

(d)

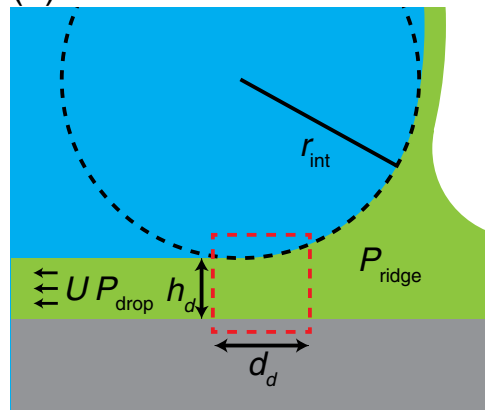

(b)

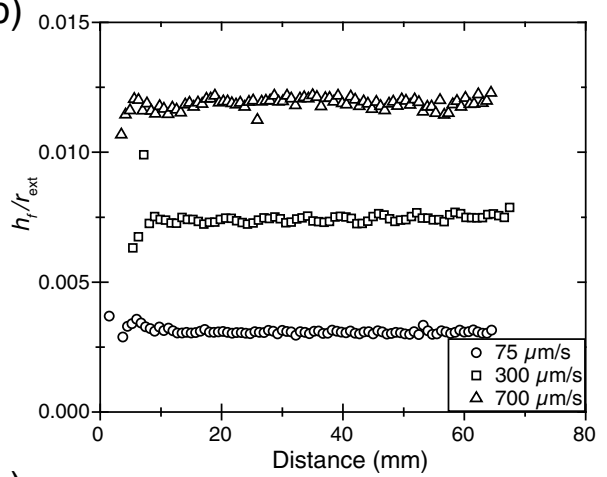

(e)

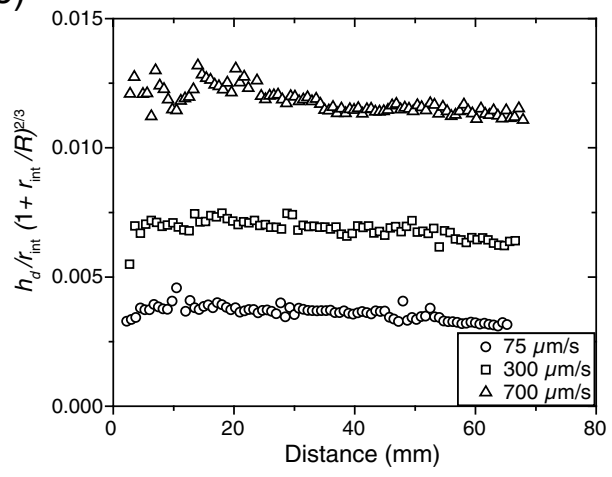

(c)

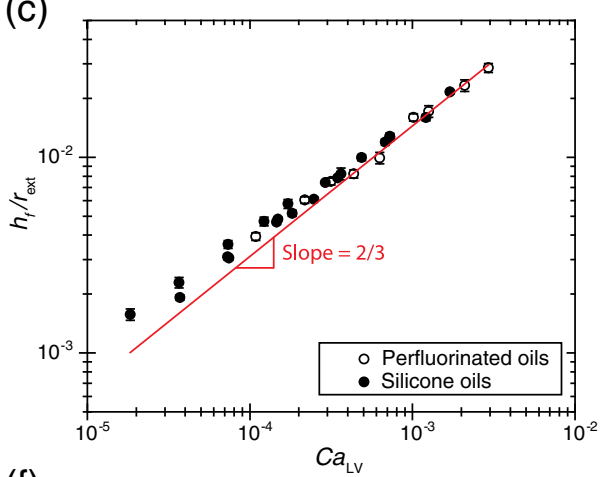

(f)

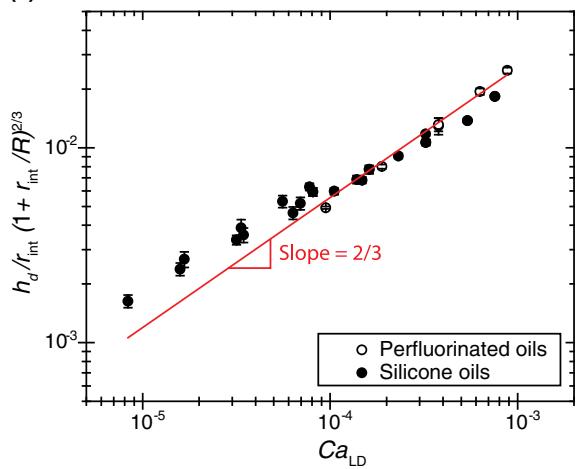

FIG. 4. (a) Schematic showing the LLD film geometry of thickness $h_{f}$ at the trail behind the moving droplet. (b) $h_{f} / r_{\mathrm{ext}}$ vs distance for droplets moving on 20-cP silicone-oil-infused surfaces under various experimental conditions. (c) Scaling of $h_{f} / r_{\mathrm{ext}}$ with $C a_{\mathrm{LV}}$ for droplets moving on surfaces infused with silicone and perfluorinated oils of various viscosities. (d) Schematic showing the LLD film geometry of thickness $h_{d}$ beneath the moving droplet. (e) $h_{d} / r_{\text {int }}\left(1+r_{\text {int }} / R\right)^{2 / 3}$ vs distance for droplets moving on 20-cP silicone-oilinfused surfaces under various experimental conditions. (f) Scaling of $h_{d} / r_{\text {int }}\left(1+r_{\text {int }} / R\right)^{2 / 3}$ with $C a_{\mathrm{LD}}$ for droplets moving on surfaces infused with silicone and perfluorinated oils of various viscosities. In all cases, the droplet volume is $25 \mu \mathrm{l}$ with initial thickness $h_{i}=4 \mu \mathrm{m}$. Data points in (c) and (f) are averages of the scaling arguments measured during the entire experiment, and error bars indicate the standard deviation. Lines of best fit are indicated in red. 
where $C a_{\mathrm{LV}}=\eta U / \gamma_{\mathrm{LV}}$ is the corresponding capillary number.

When $r_{\text {ext }} \ll R$, we recover the classical LLD results where $h_{f} / r_{\mathrm{ext}} \sim C a_{\mathrm{LV}}^{2 / 3}$; i.e., $h_{f} / r_{\mathrm{ext}}$ does not change with the distance traveled [Fig. 4(b)]. For large droplets $(V=25 \mu 1, R=2.05 \mathrm{~mm})$, the LLD classical law is well obeyed over a wide range of capillary numbers $C a_{\mathrm{LV}}=$ $10^{-5}-10^{-3}$ with perfluorinated and silicone oils used as lubricants. We observe minor deviation in the scaling behavior when $C a_{\mathrm{LV}}<10^{-4}$, likely because we neglect the finite thickness of the nanostructured surface (approximately $50-100 \mathrm{~nm}$ ), which is expected to introduce some error at low capillary numbers when $h_{f}$ is small. The red line in Fig. 4(c) shows the best-fit line with a prefactor of $\beta=1.44$, in good agreement with the numerical value obtained in the classical Landau-Levich analysis, $\beta=0.643(3)^{2 / 3} \approx 1.34$ [32].

The data in Fig. 4(c) are taken using $h_{i}=4 \mu \mathrm{m}$ and a constant drop volume of $25 \mu \mathrm{l}$ across experiments, but for a range of tested lubricant thicknesses, we find no direct dependence of $h_{f} / r_{\mathrm{ext}}$ on initial thickness. For droplets smaller than $V<10 \mu \mathrm{l}$, the effect of $R$ can no longer be ignored and Eq. (5) applies. Details of the scaling behavior observed for different initial conditions and droplet radii can be found in Appendixes E and F.

We can make a similar argument about the scaling of $h_{d}$, the thickness under the droplet, but in this case, the transition region is between the advancing wetting ridge and under the droplet, as shown in Fig. 4(d). Thus, the pressure difference is $\Delta P=P_{\text {ridge }}-P_{\text {drop }}=$ $-\gamma_{\mathrm{LD}}\left(1 / r_{\text {int }}+1 / R\right)$. Using the same arguments that we use when deriving Eq, (5), we arrive at the following result:

$$
h_{d} / r_{\text {int }}\left(1+r_{\text {int }} / R\right)^{2 / 3} \sim C a_{\mathrm{LD}}^{2 / 3},
$$

where the capillary number here is defined using the interfacial tension between the droplet and the lubricant, i.e., $C a_{\mathrm{LD}}=\eta U / \gamma_{\mathrm{LD}}$. As $r_{\text {int }}$ is difficult to measure directly, we measure the external radius of the advancing wetting ridge $r_{\text {ext }}$ and use the relation established in Eq. (3) to calculate $r_{\text {int }}$. We find that the term $h_{d} / r_{\text {int }}\left(1+r_{\text {int }} / R\right)^{2 / 3}$ is constant throughout the distance traveled in a given experiment [Fig. 4(e)]. We see that the scaling behavior follows Eq. (6), as shown in Fig. 4(f). The prefactor in this case is 2.58, which differs substantially from that in classical LLD analysis, since we ignore the three-dimensional nature of the fluid flow at the droplet base [33]. The deviation between silicone and perfluorinated oils in Fig. 4(f) at high capillary numbers is likely due to the difficulty in aligning the optical probe when the droplet is moving at high speeds. Minor deviations at low speeds correspond to the finite size of the nanostructures, as discussed earlier.

Note that Eq. (6) is slightly different from the scaling reported by Daniel et al., where the effect of the wetting ridge on the droplet geometry was neglected, and it was assumed that $h_{d} / R \sim C a_{\mathrm{LD}}^{2 / 3}$ [18]. Importantly, this discrepancy does not change the scaling law for the dissipation force on a moving droplet reported in that paper.

A qualitatively similar framework can be applied to the dynamic thickness of the lubricant wrapping layer $h_{w}$. For a static droplet at equilibrium, $h_{w}$ is stabilized by van der Waals interactions and is typically tens of nanometers thick (Appendix G, Fig. 12). A moving droplet, however, will entrain a lubricant film with it, and $h_{w}$ thickens with increasing velocity. A strict LLD analysis, however, no longer holds because of the spherical geometry of the droplet. This geometry gives rise to a complex 3D fluid flow and a resulting wrapping layer that is nonuniform in thickness, which can be visualized using color-interferometric techniques [34]. We illuminate the droplet using diffuse white lighting; the local film thickness can now be deduced from the ensuing interference patterns captured using a digital camera [Fig. 5(a)]. Droplet motion results in a complex lubricant profile. Notably, the lubricant tends to wrap around the sides, forming an extended skirt above the wetting ridge; the lubricant is thicker just above the wetting ridge but becomes much thinner towards the top of the droplet. Additionally, as compared to the lubricant under and behind a moving droplet, we see much more irregularity in the thickness of the wrapping film, possibly due to the complex interaction between the droplet's internal flow, the lubricant flow, and the draft in ambient air (Fig. 12 and Supplemental Material Video S2 in [27]).

This technique can be used to quantitatively describe the profile of a thin film, since each color corresponds to a specific film thickness [34]; it is difficult, however, to distinguish between thicknesses above $500 \mathrm{~nm}$ because of the overlap in the color scale [see, for example, the reference colors in Fig. 5(a)]. Hence, to quantitatively evaluate the extent to which LLD can be used to describe $h_{w}$, we choose to utilize white-light interference measurements using a spectrometer, as before. The size of the optical probe prevents us from placing it behind the droplet, where the assumptions of LLD are more valid. Hence, we position the probe at the side of the droplet, where it is flattest [marked yellow on Fig. 5(b)]. Note that because of the poor refractive-index contrast and higher variability in thickness, the minimum $h_{w}$ that can be accurately measured in this configuration is approximately $400 \mathrm{~nm}$, which prevents us from measuring the thickness early in an experiment or at lower capillary numbers.

Because of the complications described above, we do not expect the LLD framework to fully describe $h_{w}$; however, we do expect some similarities. We follow the analysis used for $h_{f}$ and $h_{d}$ to determine the scaling behavior. In this case, the pressure difference in the transition region-between the wetting ridge and the lubricant in the wrapping layeris $\Delta P=P_{\text {ridge }}-P_{\text {wrapping }}=-\gamma_{\mathrm{LV}}\left(1 / r_{\text {ext }}+1 / R\right)$, resulting in the equation 
(a)

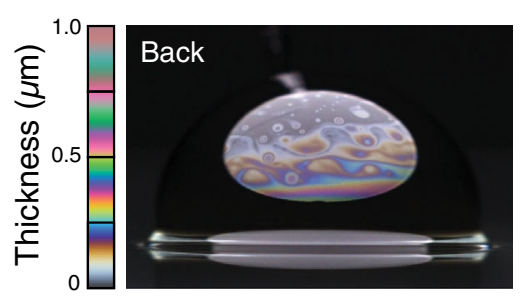

(b)

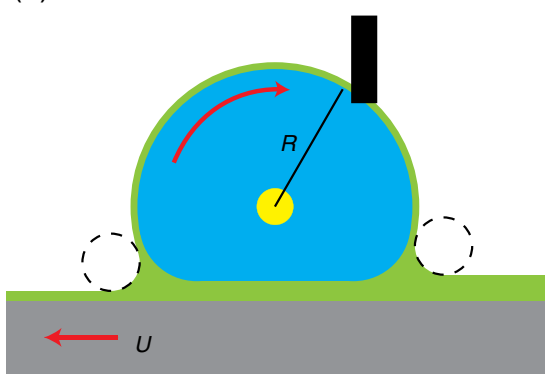

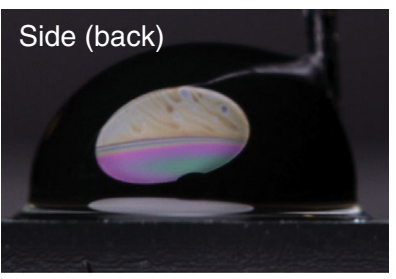
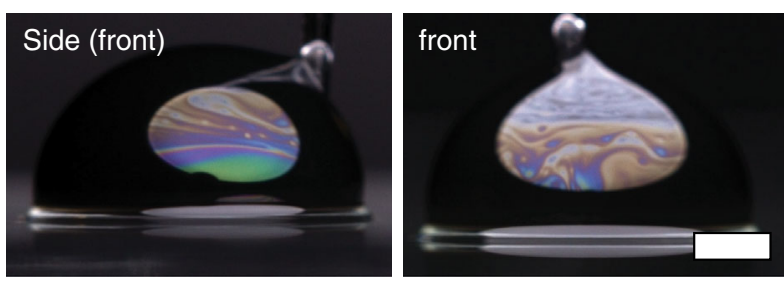

(c)

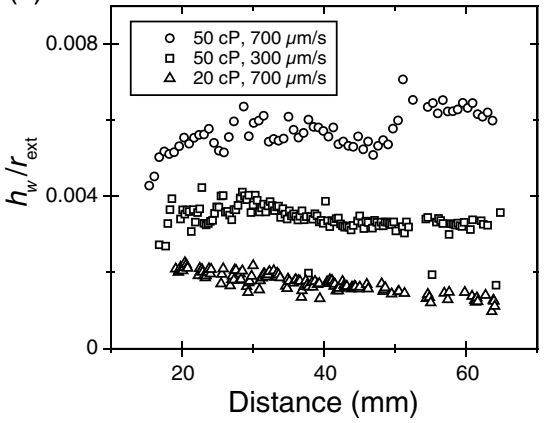

(d)

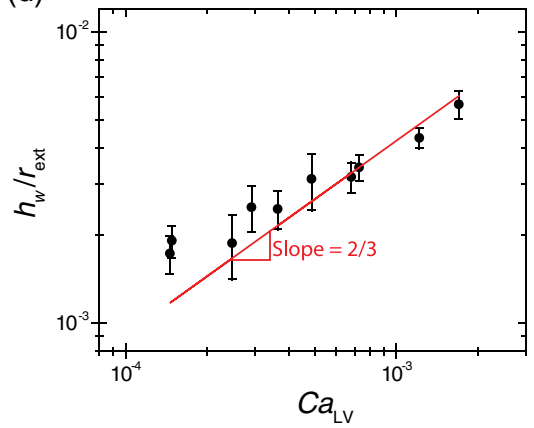

FIG. 5. (a) The wrapping layer imaged using color interferometry at different positions on the surface of a moving droplet. Note, the complex lubricant dynamics limit the extent to which LLD can be applied to $h_{w}$. The thickness scale on the left is calculated computationally and should be used only as a guide. $V_{\text {drop }}=25 \mu \mathrm{l}, \mu=50 \mathrm{cP}, U=150 \mu \mathrm{m} / \mathrm{s}, h_{i}=4 \mu \mathrm{m}$, and $L=40 \mathrm{~mm}$. Scale $\mathrm{bar}=1 \mathrm{~mm}$. Note, the "side (front)" image is reflected to match the orientation of the other images. (b) Experimental schematic. The yellow dot indicates the position of the optical probe used to measure $h_{w}$. (c) $h_{w} / r_{\mathrm{ext}}$ as a function of the position for different capillary number experiments. (d) Scaling of $h_{w} / r_{\mathrm{ext}}$ with $\mathrm{Ca} a_{\mathrm{LV}}$. The line of the best fit is indicated in red.

$$
h_{w} / r_{\mathrm{ext}}\left(1+r_{\mathrm{ext}} / R\right)^{2 / 3} \sim C a_{\mathrm{LV}}^{2 / 3},
$$

which simplifies to $h_{w} / r_{\mathrm{ext}} \sim C a_{\mathrm{LV}}^{2 / 3}$ when the wetting ridge is much smaller than the dimensions of the droplet.

We find that $h_{w} / r_{\mathrm{ext}}$ is relatively constant for a given experiment, although we observe increased noise, particularly at high capillary numbers, due to the variability in $h_{w}$ and more challenging probe alignment [Fig. 5(c)]. We experimentally determine the prefactor $\beta=0.42$ for the scaling of $h_{w} / r_{\text {ext }}$ with $C a^{2 / 3}$ [Fig. 5(d)]. Not surprisingly, the fit is relatively poor, and the prefactor differs significantly from the classical Landau-Levich prefactor $\beta \approx 1.34$, which was developed for two-dimensional flow. A full description of lubricant dynamics in the wrapping layer is a rich and challenging problem and is outside the scope of this study.

It has been suggested that the presence of the wrapping layer is the major contribution to lubricant depletion [22]. In our experiments, the maximum value of $h_{w}$ measured at the highest capillary number is approximately $1.5 \mu \mathrm{m}$. Applying this value to the total droplet's surface-which greatly overestimates the amount of lubricant in the wrapping layer-results in a total volume that is about an order of magnitude smaller than the volume of lubricant in the wetting ridge (tens of nanoliters as opposed to hundreds). The wrapping layer is, therefore, a minor consideration in the overall depletion of the lubricant overlayer; a much more important source of lubricant depletion is the growth of the wetting ridge, which we discuss in the next section.

\section{Lubricant depletion}

In Sec. II B, we describe how the various lubricant dynamics and the resultant film thicknesses $\left(h_{f}, h_{d}\right.$, and $\left.h_{w}\right)$ can be understood in terms of the classical LLD problem. Here, we explain how lubricant depletion arises from the interconnected lubricant dynamics and how it is intimately linked to the growth of the wetting ridge. In particular, we explicitly show that the volume of the wetting ridge $V_{\text {ridge }}$ is equal to the volume of lubricant being depleted $V_{\text {lost }}$ due to the change in thicknesses $\Delta h=h_{i}-h_{f}$. With this physical insight, we are then able to describe the process of lubricant depletion in SLIPS fully.

First, we note that

$$
V_{\text {ridge }}=\alpha 2 \pi a r_{\mathrm{ext}}^{2},
$$

where $\alpha$ is a geometric factor to account for the exact shape. The exact value of $\alpha$ can change as the wetting ridge grows in size and can depend on $\mathrm{Ca}$; nevertheless, $\alpha$ should remain at about 0.5 , since the wetting ridge can be approximated in the first instance as a triangle.

To calculate $V_{\text {lost }}$, we need to know the profile of lubricant trail behind a moving droplet, which we deduce using RICM [Fig. 6(a)]. Along the midline, we show how $h_{f}$ follows the LLD scaling law [Eq. (5)]. Away from the 
(a)

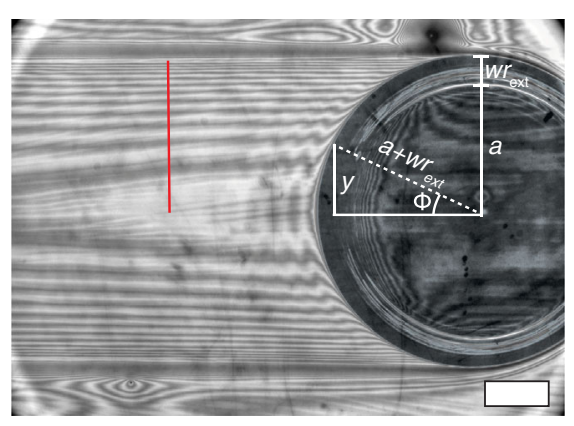

(b)

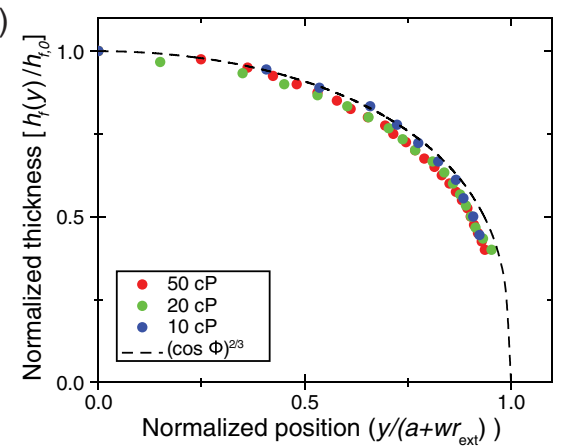

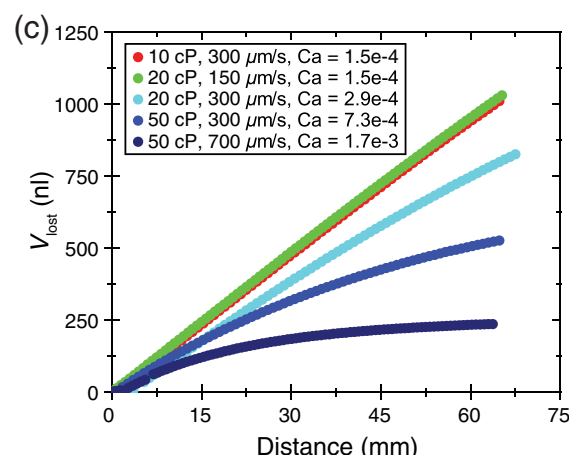

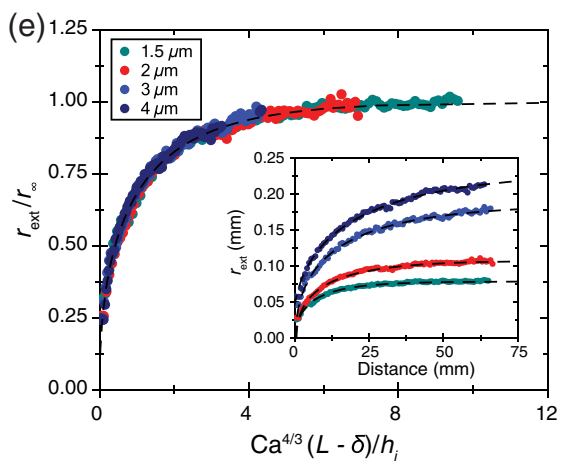

(d)

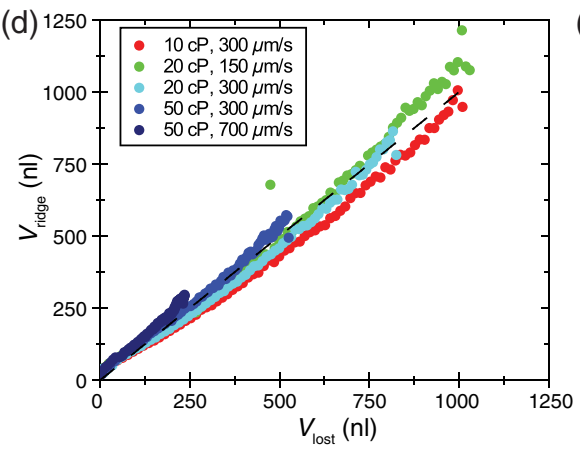

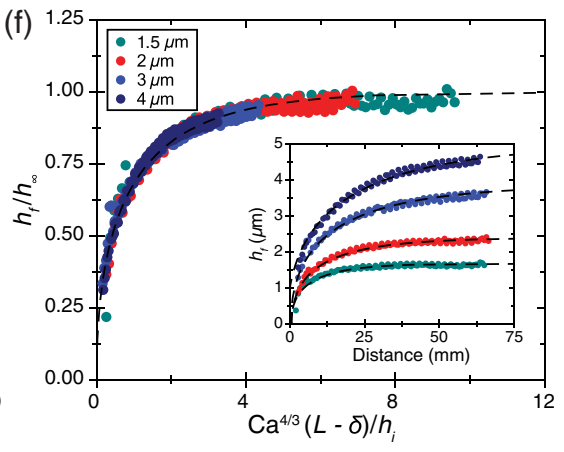

FIG. 6. (a) RICM of a droplet moving on SLIPS, including the geometric components used to describe the shape of the lubricant behind the droplet (scale bar $=1 \mathrm{~mm}$ ). (b) Normalized thickness profiles behind the droplet calculated using RICM intensity profiles [for example, along the red line in (a)] in combination with white-light interference measurements. In all cases, $h_{i}=4 \mu \mathrm{m}, V=25 \mu \mathrm{l}$, and $U=300 \mu \mathrm{m} / \mathrm{s}$. (c) $V_{\text {lost }}$ vs distance for droplets moving with various capillary numbers over silicone oil SLIPS with $h_{i}=4 \mu \mathrm{m}$. (d) $V_{\text {ridge }}$ vs $V_{\text {lost }}$ for experiments on silicone oil with various capillary numbers. The sole fitting parameter $\alpha=0.52$. (e),(f) Nondimensional plots of the growth of $r_{\text {ext }}$ and $h_{f, 0}$ with distance for droplets moving at $700 \mu \mathrm{m} / \mathrm{s}$ on 50-cP silicone oil with various initial thicknesses (insets show dimensional quantities). Dashed lines indicate best-fit lines predicted by Eq. (10).

midline along $y$, we find that the thickness of the lubricant film $h_{f}(y)$ can be described by a modified LLD scaling law $h_{f} / r_{\text {ext }} \sim\left(\eta U_{\phi} / \gamma_{\mathrm{LV}}\right)^{2 / 3}$, where $U_{\phi}=U \cos \phi$ is the radial component of the velocity. Equivalently, $h_{f}(y) / h_{f, 0}=$ $(\cos \phi)^{2 / 3}$, where $h_{f, 0}$ is the film thickness at $y=0$ as described by Eq. (5).

Although single-wavelength RICM can give only information on the relative lubricant thicknesses at different regions, combining RICM with white-light interferometry measurements allows us to deduce the thickness profile $h_{f}(y)$ unambiguously. For lubricants of different viscosities $\eta=10-50 \mathrm{cP}$, we find a maximum lubricant thickness at $y=0$, which then decreases monotonically towards the edges of the droplet following the modified LLD scaling law described above [dashed line in Fig. 6(b)]. From the profile, we can integrate numerically to calculate the average thickness behind the droplet, resulting in $\left\langle h_{f}\right\rangle \approx$ $0.85 h_{f, 0}=0.85 \beta r_{\mathrm{ext}} C a_{\mathrm{LV}}^{2 / 3}$.

Once $r_{\text {ext }}(L)$ and $h_{f, 0}(L)$ [and, hence, $\left\langle h_{f}(L)\right\rangle$ ] are determined experimentally, the amount of lubricant loss $V_{\text {lost }}(L)$ can then be calculated numerically:

$$
V_{\text {lost }}(L)=\int_{0}^{L} 2\left(a+w r_{\mathrm{ext}}\right) \Delta h d L+V_{o},
$$

where $L$ is the distance traveled by the droplet, $\Delta h=$ $h_{i}-\left\langle h_{f}\right\rangle, w$ is a geometric factor to account for the added width of the wetting ridge, and $V_{0}=\alpha 2 \pi a r_{\text {ext, } 0}^{2}$ is the volume of the wetting ridge of size $r_{\text {ext, } 0}$ created when the droplet is first placed on the surface.

Figure 6(c) shows the progression of $V_{\text {lost }}(L)$ for droplets moving with different $C a_{\mathrm{LV}}$ and the same $h_{i}=4 \mu \mathrm{m}$. We note that droplets with higher capillary numbers deplete much less lubricant than slower-moving droplets on less viscous lubricants, and $V_{\text {lost }}$ appears to plateau to a maximum value for the highest $C a_{\mathrm{LV}}=1.7 \times 10^{-3}$-an observation which we explain later. We also find that the growth of the wetting ridge $V_{\text {ridge }}(L)$ follows that of $V_{\text {lost }}(L)$, as shown in Fig. 6(d). The only fitting parameter here is $\alpha_{\text {fit }}=0.52$, close to the expected value in our analysis [Eq. (8)]. The curvature observed in Fig. 6(d) indicates that while $\alpha$ does not vary greatly, it decreases with increasing wetting ridge size and increasing $C a$.

We can also directly predict the lubricant loss $V_{\text {lost }}(L)$ by first solving for $r_{\mathrm{ext}}(L)$, which follows the differential equation $\quad \alpha 4 \pi a r_{\text {ext }}\left(d r_{\text {ext }} / d L\right)=d V_{\text {lost }} / d L=2\left(a+w r_{\text {ext }}\right)$ $\Delta h \approx 2 a \Delta h$. Replacing $\Delta h$ with the appropriate scaling law, as discussed earlier, this equation can be integrated and rearranged to give 


$$
\ln \left(1-\frac{r_{\mathrm{ext}}}{r_{\infty}}\right)+\frac{r_{\mathrm{ext}}}{r_{\infty}}=-\mu_{1} C a_{\mathrm{LV}}^{4 / 3}\left(\frac{L-\delta}{h_{i}}\right)
$$

where $\mu_{1}=\chi^{2} \beta^{2} /(2 \pi \alpha) \approx 0.5, \chi=\left\langle h_{f}\right\rangle / h_{f, 0} \approx 0.85$, and $r_{\infty}=h_{i} / \chi \beta C a_{\mathrm{LV}}^{2 / 3}$ is the limiting size of the wetting ridge for a given set of experimental conditions. The integration constant $\delta$ is a small positional offset to account for the experimental error in $L$ and the initial wetting ridge size $r_{\mathrm{ext}, 0}$.

Figure 6(e) shows the growth of $r_{\text {ext }}(L)$ for droplets moving at $0.7 \mathrm{~mm} / \mathrm{s}$ on surfaces with different initial lubricant thicknesses $h_{i}=1.5-4 \mu \mathrm{m}$ of 50-cP silicone oil. As predicted by Eq. (10), the data collapse to a single curve on a nondimensional plot of $r_{\mathrm{ext}} / r_{\infty}$ against $C a_{\mathrm{LV}}^{4 / 3}(L-\delta) / h_{i}$. The dashed lines are the corresponding best-fit curves with $\alpha, \delta$, and $\chi$ as fitting parameters. The fitted values are consistent with our previous analysis: $\alpha_{\text {fit }}=0.51 \pm 0.05 ;\left|\delta_{\text {fit }}\right|$ is $<1 \mathrm{~mm}$, much smaller than the total length traveled $L>6 \mathrm{~cm}$, indicating that the error in $L$ and $r_{\text {ext, } 0}$ is minimal, and $\chi_{\text {fit }}=0.80 \pm 0.01$, close to the expected value of 0.85 . The slight discrepancy in the fitted value of $\chi$ could be caused by the change in the depleted path due to the finite size of the wetting ridge, which we ignore when deriving Eq. (10). Details of the numerical fit can be found in Appendix H, Table IV.

An analytic solution also exists in the limit that $r_{\text {ext }} /$ $r_{\infty} \ll 1$, whereby $\ln \left(1-r_{\text {ext }} / r_{\infty}\right)+r_{\text {ext }} / r_{\infty} \approx-1 / 2$ $\left(r_{\text {ext }} / r_{\infty}\right)^{2}$, and Eq. (10) simplifies to

$$
r_{\mathrm{ext}}=\sqrt{\frac{h_{i}(L-\delta)}{\pi \alpha}}
$$

i.e., the growth of the wetting ridge is initially independent of the droplet's speed, and fresh drops cause significant local depletion regardless of capillary number. Equation (11) can also be derived by noting that in this limit, $\Delta h \approx h_{i}$ and, hence, $d V_{\text {lost }} / d L \approx 2 a h_{i}$.

Once $r_{\text {ext }}(L)$ is known, $h_{f, 0}(L)$ can be found trivially by applying LLD law [dashed lines in Fig. 6(f), where $\left.h_{\infty}=\beta r_{\infty} C a_{\mathrm{LV}}^{2 / 3}\right]$ and $V_{\text {lost }}(L)$ then follows from Eq. (9). Note that as $r_{\text {ext }}$ increases, the Laplace pressure decreases, and the rate of lubricant depletion decreases until it reaches zero when $\Delta h=0$ and, hence, $d V_{\text {lost }} / d L=0$; this limit is reached when $h_{f, 0}=h_{\infty}$ and $r_{\mathrm{ext}}=r_{\infty}$. The maximum amount of lubricant loss can then be deduced by noting that $V_{\text {lost,max }}=V_{\text {ridge,max }}$, i.e.,

$$
V_{\text {lost, } \max }=\alpha 2 \pi a r_{\infty}^{2}=\frac{a h_{i}^{2}}{\mu_{1} C a_{\mathrm{LV}}^{4 / 3}} .
$$

While we explicitly demonstrate only lubricant depletion due to water droplets, the results presented here, such as Eq. (12), can be applied to other liquid droplets (including for small $\left.\theta_{\text {app }}\right)$ as long as $r_{\text {ext }} \ll R$.

This framework leads us to several conclusions, some of which run against intuitive expectations. Equation (12) shows that droplets moving at higher velocities and capillary numbers cause less lubricant to be lost. From previous work, we know that the drag force for a droplet moving across SLIPS scales with $F_{d} \sim \gamma R C a^{2 / 3}[18,31]$. Counterintuitively, this means that droplets moving across the surface with a higher driving force and correspondingly higher shear rate deplete less lubricant than slower-moving droplets.

We can further consider how the size of the droplet affects lubricant loss. We note that $V_{\text {lost }}$ scales with $a \sim R$, while droplet volume scales with $R^{3}$. Thus, to minimize depletion, it is advantageous to have one large droplet rather than several smaller droplets of the same total volume. For applications such as condensation where water initially forms on the surface as small, discrete droplets, strategies that promote rapid coalescence into bigger droplets can lead to improved longevity [35].

In our paper, we make extensive use of LLD analysis, which is known to break down for large $C a>10^{-2}$ [36-39]. However, we can expect the lubricant thickness to scale as $h \sim r C a^{\nu}$, where $\nu$ is some exponent which differs from 2/3 [39-41]. Consequently, many of the results discussed here can still be applied with some modifications. For example, the amount of lubricant loss can be generalized to $V_{\text {lost,max }}=a h_{i}^{2} / \mu_{1} C a_{\mathrm{LV}}^{2 \nu}$ [Eq. (12)]. Our preliminary results indicate that $\nu \approx 1 / 4$ for $\mathrm{Ca}>10^{-2}$; its exact value (and its derivation) is still an open question and beyond the scope of this study (Appendix I, Fig. 13).

The amount of lubricant lost scales with $h_{i}^{2}$. As lubricant is depleted, less and less volume is lost, with little impact on surface performance, since as we showed in earlier work, $F_{d}$ is independent of $h_{i}$ [18]. This behavior remains unchanged until the lubricant thickness becomes nanometric in size, when dispersion forces (such as van der Waals interactions) must be considered. Consideration of this regime presents a natural opportunity for extension of this work in the future.

Finally, we use only nanostructured surfaces, where the length scale of the structures is significantly smaller than the micron-scale lubricant thicknesses. The scaling relationships derived in this work also applies directly to flat surfaces, such as liquid-infused organogels $[42,43]$ but must be modified for microstructured surfaces. However, the main idea outlined in this work-that the wetting ridge is a lowpressure region and its growth drives lubricant depletion-is general and likely to remain true for most surfaces.

\section{CONCLUSIONS}

Our work here can be split into three parts. In part I, we show that the geometry of a droplet on SLIPS can be understood by balancing Laplace pressure and using geometric arguments. In part II, we show how the various lubricant thicknesses change dynamically with speed and can be deduced-analogous to the classical LandauLevich-Derjaguin problem-by balancing the pressure 
gradient and viscous stress at the edge of the wetting ridge. Finally, in part III, we use this understanding to identify the growth of the wetting ridge as the main source of lubricant depletion and to quantify the amount of lubricant that a moving droplet collects as it sweeps across a lubricantinfused surface. While we explicitly discuss only lubricant depletion due to water droplet motion, many of the ideas explored here will be useful in understanding lubricant depletion by other droplets and in very different situations, such as during ice formation and droplet condensation on lubricated surfaces. By identifying the main source and mechanics of lubricant loss on SLIPS, our work will inform the design of longer-lasting lubricant-infused surfaces.

\section{MATERIALS AND METHODS}

Boehmite synthesis: All SLIPS used in this work are created using transparent thin films of nanostructured boehmite on glass [15]. Briefly, an alumina sol-gel solution is spin coated onto $3 \times 1 \mathrm{in}^{2}$ glass microscope slides at $1000 \mathrm{rpm}$ and dried at $70^{\circ} \mathrm{C}$ for $1 \mathrm{~h}$. Boehmite is formed from the alumina sol-gel by submersion in deionized (DI) water for $30 \mathrm{~min}$ at $60^{\circ} \mathrm{C}$. The surfaces are rinsed with DI water and then blown dry with nitrogen.

Surface functionalization: For SLIPS infused with a silicone oil as a lubricant, the nanostructured sample is placed in a sealed jar with a small piece of cured 10:1 Sylgard 184 Silicone Elastomer and heated at $235^{\circ} \mathrm{C}$ for $7 \mathrm{~h}$ [44]. The samples are then rinsed with ethanol and dried with nitrogen before application of silicone oil. SLIPS samples infused with perfluorinated polyether oils are first functionalized using perfluoroalkyl phosphate ester (FS100 surfactant). Boehmite-coated glass slides are submerged in a solution of $95: 5: 1$ by weight ethanol:DI water:FS100 for $1 \mathrm{~h}$ at $70^{\circ} \mathrm{C}$. The samples are then rinsed thoroughly with acetone, ethanol, and IPA and blown dry using nitrogen before application of perfluoropolyether oils.

Lubricant application: Silicone oils purchased from Sigma Aldrich with viscosities in the range of 5-50 cP are used. The interfacial tensions (IFTs), as measured using the pendant droplet method, are roughly $19 \mathrm{mN} / \mathrm{m}$ in air and $42 \mathrm{mN} / \mathrm{m}$ in water, with minor variations due to viscosity. Two perfluoropolyethers are used with viscosities of 23.2 and $72.6 \mathrm{cP}$ (Dupont Krytox GPL 100 and 102, respectively). The IFT of the Krytox oils is $16 \mathrm{mN} / \mathrm{m}$ in air and $58 \mathrm{mN} / \mathrm{m}$ in water, again with minor variation with viscosity. The lubricants are applied by spin coating at defined speeds, and the film thickness is measured using white-light interferometry.

Dyed water: Black dyed water is used in order to minimize optical reflections during measurements. A thick layer of soot is created on a clean glass Petri dish by placing it over a candle flame. The soot is hydrophilized by exposure to oxygen plasma for $5 \mathrm{~min}$, dissolved in pure deionized millipore water, and filtered through a $0.45-\mu \mathrm{m}$ cellulose filter. The resulting solution is used as a stock solution that is then diluted 5:1 for all measurements and experiments. The IFT of the dyed droplet in air and with oil is measured using the pendant drop method and found to differ from that of pure water by less than $1 \%$.

Wetting ridge measurement: A digital camera (Panasonic GH4) is calibrated for scale and used to take pictures of the wetting ridge profile at a rate of $1 \mathrm{frame} / \mathrm{sec}$. The point at which the wetting ridge meets the horizontal surface and the point where the wetting ridge meets the droplet are tracked in 2D space using an open-source tracking software [45]. The wetting ridge radius is calculated as the average of the difference in the $x$ and $y$ coordinates of these two points. In cases where the wetting ridge is discontinuous with the surface of the droplet, the radius is calculated as $\left(\Delta x^{2}+\Delta y^{2}\right) / 2 \Delta y$, where $\Delta x$ and $\Delta y$ are the differences in the $x$ and $y$ coordinates of the two points.

Dynamic thickness measurements: The thickness measurements are performed using an Ocean Optics USB2000 + spectrometer with a halogen lamp as the white-light source. A reflectance-mode optical fiber is placed under the sample, and immersion oil is placed between the glass and the probe to eliminate reflection from the back of the glass slide. The spectrum is normalized against the light source, resulting in a series of peaks and valleys appearing due to the difference in path length between the lubricant-substrate and lubricant-air or -water interfaces. A reliable spectroscopic signal can be obtained with integration times of around $20 \mathrm{~ms}$ (equivalent to about $50 \mathrm{fps}$ ). By analyzing the wavelengths of the interference maxima and minima, the thickness of the thin films is unambiguously determined in a range from $400 \mathrm{~nm}$ to several microns, as described previously [18].

Reflection interference contrast microscopy: The samples are imaged with a custom inverted microscope in reflection mode. Monochromatic light is produced by passing broadband LED illumination through a 532-nm filter. Thus, two adjacent maxima or minima differ in thickness by $\lambda / 2 n_{\text {lub }}$ or 0 , and assumptions about the shape or initial thickness must be made in order to obtain a quantitative thickness profile. For our RICM measurements, we take images at $1 \mathrm{fps}$ with an exposure time of roughly $1 / 30-1 / 60 \mathrm{~s}$. The same setup can easily be used to take video at up to $60 \mathrm{fps}$. With a high-speed camera, much higher frame rates are possible, with the main limitation being the intensity of the LED light source (which can be replaced with brighter sources as needed). Typically, such high speeds are not necessary for the phenomena investigated in this study.

Fluorescence confocal imaging: Confocal imaging is done using a Zeiss LSM 700 upright confocal with a $40 \times$ water immersion objective, and 20-cP silicone oil is dyed with $2.5 \%$ by volume of DFSB-K175 fluorescent dye to generate a fluorescence signal. Further details can be found in Appendix A. 


\section{ACKNOWLEDGMENTS}

The work is supported partially by the Office of Naval Research, U.S. Department of Defense, under Grant No. N00014-17-1-2913 and by the Advanced Research Projects Agency-Energy, U.S. Department of Energy, under Award No. DE-AR0000326. J. V. I. T. is supported by the European Commission through the Seventh Framework Programme project DynaSLIPS (Project No. 626954). We acknowledge the use of the facilities at the Harvard Center for Nanoscale Systems supported by the NSF under Grant No. ECS-0335765. M. J. K. thanks the Natural Sciences and Engineering Research Council of Canada for a PGS-D scholarship.

M. J. K. and D. D. contributed equally to this work.

\section{APPENDIX A: DETAILS OF CONFOCAL MICROSCOPY STUDY}

To verify our geometric model of a droplet on SLIPS, we dye $20-\mathrm{cP}$ silicone oil with $2.5 \%$ by volume DFSB-K175 fluorescent dye and create a fluorescent $z$-stack image of the wetting ridge using a confocal microscope, allowing for the measurement of $r_{\text {int }}$ and $r_{\text {ext }}$. The dye is excited using a laser wavelength of $405 \mathrm{~nm}$, and the fluorescent signal is collected in the range of 560-800 nm. Confocal imaging is done using a Zeiss LSM 700 upright confocal with a $40 \times$ water immersion objective. The wetting ridge is imaged through the base of the SLIPS sample, which is nanostructured boehmite coated onto a 1-mm-thick glass slide. As there is a mismatch between the refractive index of the silicone oil and the water immersion objective, the $z$ height is scaled by the factor $n_{\text {oil }} / n_{\text {water }}$ as discussed by Besseling et al. [46]. The interfacial tension of the fluorescent lubricant is measured in air and water (19 and $35 \mathrm{mN} / \mathrm{m}$, respectively) using the pendant drop method.

Each slice of the $z$ stack is radially averaged along the circumference of the wetting ridge and then stacked to generate a $2 \mathrm{D}$ intensity map of the wetting ridge cross section. The profile of the wetting ridge is determined using a combination of intensity thresholding and gradient analysis, and circular fitting is used to calculate the radii $r_{\text {ext }}$ and $r_{\text {int }}$ [Fig. 1(b) in the main text].

We image the wetting ridge around droplets of 3 and $8 \mu \mathrm{l}$, which are small enough that the inverted surface orientation and gravity can be ignored. The results of the analysis are summarized in Table I.

TABLE I. Geometric values obtained through confocal analysis.

\begin{tabular}{lccc}
\hline \hline Volume $(\mu \mathrm{l})$ & $a \sim R(\mu \mathrm{m})$ & $r_{\mathrm{ext}}(\mu \mathrm{m})$ & $r_{\mathrm{int}}(\mu \mathrm{m})$ \\
\hline 3 & 868 & 80 & 114 \\
8 & 1225 & 69 & 107 \\
\hline \hline
\end{tabular}

TABLE II. Comparison of measured and predicted $r_{\text {int }}$.

\begin{tabular}{lccc}
\hline \hline Volume $(\mu \mathrm{l})$ & $r_{\text {int,exp }}(\mu \mathrm{m})$ & $r_{\text {int,pred }}(\mu \mathrm{m})$ & Error on $r_{\text {int }}$ \\
\hline 3 & 114 & 117 & $-2.8 \%$ \\
8 & 107 & 110 & $-2.4 \%$ \\
\hline \hline
\end{tabular}

Values of $r_{\text {int }}$ predicted by Eq. (3) in the main text differ less than $3 \%$ from experimental values, as shown in Table II.

\section{APPENDIX B: DERIVATION OF $\theta_{\text {app }}$ AND MODIFIED YOUNG's EQUATION}

As we discuss in the main text, $\theta_{\text {app }}$ can be derived by balancing forces due to the interfacial tensions at the ridge, similar to argument originally proposed by Young [24] (Fig. 7).

Alternatively, $\theta_{\text {app }}$ can be deduced using geometrical arguments by requiring that the two interfaces (lubricantvapor and lubricant-droplet) of the wetting ridge are tangential to the circle of radius $R$, giving $\sin \left(\theta_{\text {app }}-\pi / 2\right)=$ $\left(r_{\text {int }}-r_{\text {ext }}\right) /\left(r_{\text {int }}+r_{\text {ext }}\right)$ or $\cos \theta_{\text {app }}=(1-\epsilon) /(1+\epsilon)$, where $\epsilon=r_{\text {int }} / r_{\text {ext }}$ [Figs. 8(a) and 8(b)]. In the limit where $r_{\text {ext }} \ll R$, Eq. (3) in the main text simplifies to $\gamma_{\mathrm{LD}} / r_{\text {int }}=$ $\gamma_{\mathrm{LV}} / r_{\mathrm{ext}}$ and, hence, $\epsilon=\gamma_{\mathrm{LD}} / \gamma_{\mathrm{LV}}$ and

$$
\cos \theta_{\mathrm{app}}=\frac{\gamma_{\mathrm{LV}}-\gamma_{\mathrm{LD}}}{\gamma_{\mathrm{LV}}+\gamma_{\mathrm{LD}}}
$$

A similar analysis can be performed in the absence of a wrapping layer, that is, $S_{\mathrm{LD}}<0$ [Figs. 8(c) and 8(d)]. Balancing Laplace pressures across interfaces gives $\gamma_{\mathrm{LD}} / r_{\text {int }}=\gamma_{\mathrm{LV}} / r_{\mathrm{ext}}+\left(2 \gamma_{\mathrm{DV}}-\gamma_{l v}-\gamma_{\mathrm{LD}}\right) / R$ [cf. Eq. (3) in the main text]. Rather than requiring a common tangent where the wetting ridge meets the droplet, there is now a three-phase contact line, where the three surface tension vectors must form a Neumann triangle, i.e., $\vec{\gamma}_{\mathrm{DV}}+$ $\vec{\gamma}_{\mathrm{LV}}+\vec{\gamma}_{\mathrm{LD}}=0$ [19-21,47]. Here, the line connecting the centers of the two circles has length of $r_{\text {int }}+r_{\mathrm{ext}}-d$, where $d$ is the size of the overlap region that can be

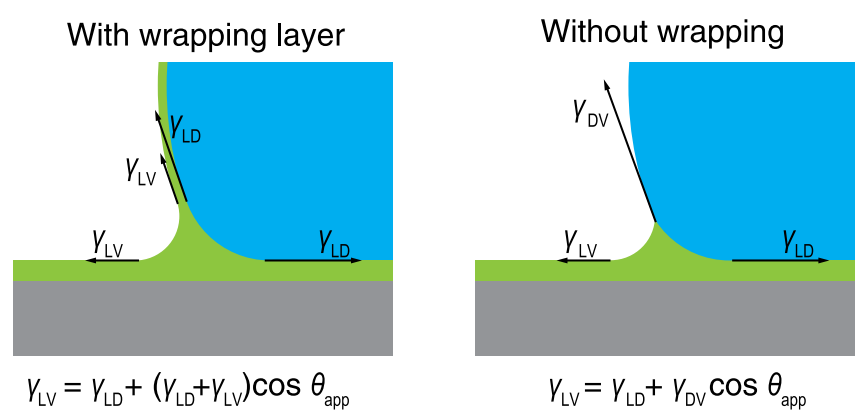

FIG. 7. The apparent contact angle $\theta_{\text {app }}$ given by a force balance of solely liquid-liquid and liquid-vapor interfacial tensions. 

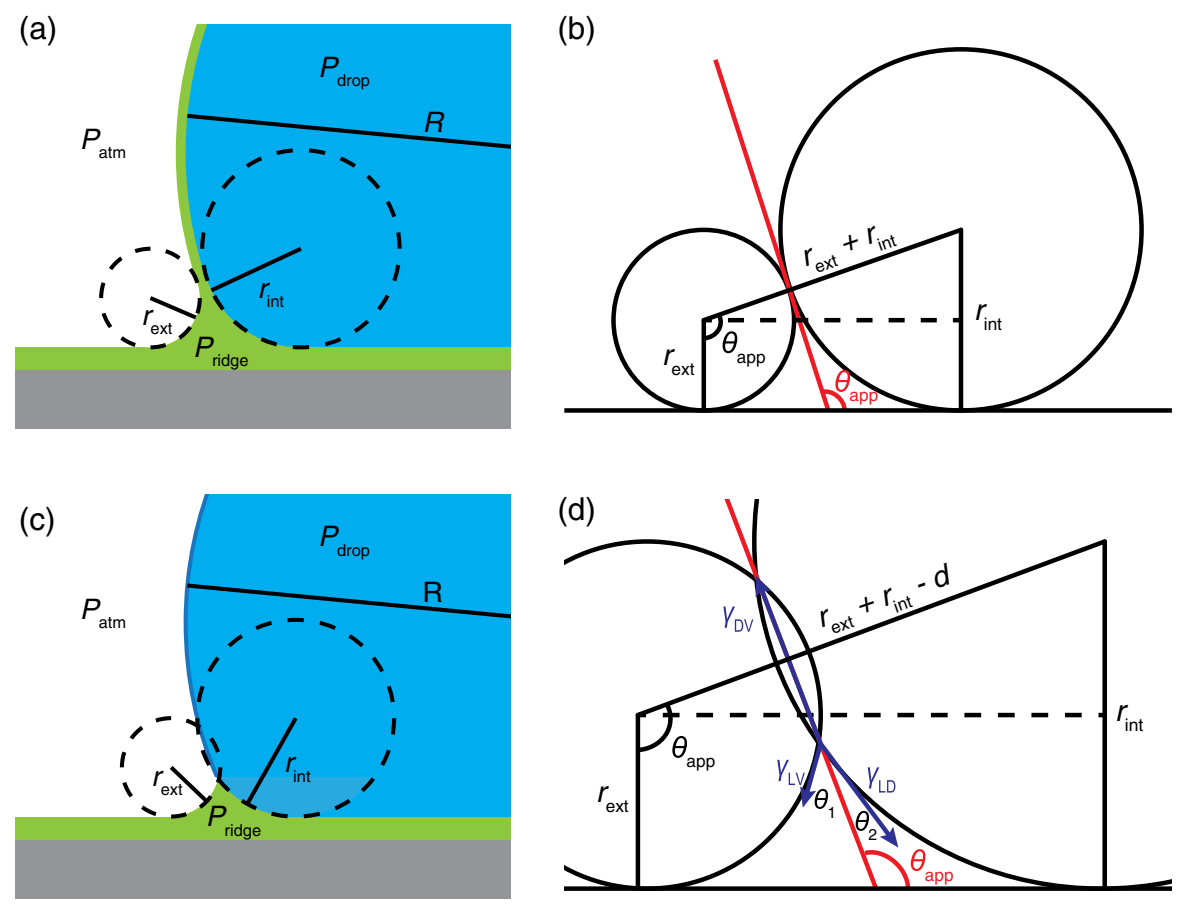

FIG. 8. (a) Schematic showing the geometry of a droplet when a wrapping layer is present $\left(S_{\mathrm{LD}}>0\right)$. (b) Geometric construction used to calculate $\theta_{\text {app }}$ when $S_{\mathrm{LD}}>0$. (c) Schematic of a droplet without a wrapping layer $\left(S_{\mathrm{LD}}<0\right)$. (d) Geometric construction used to calculate $\theta_{\text {app }}$ when $S_{\mathrm{LD}}<0$.

deduced using the geometry of circular segments, i.e., $d=r_{\text {int }}\left(1-\cos \theta_{2}\right)+r_{\text {ext }}\left(1-\cos \theta_{1}\right)$. Hence, $r_{\text {int }}+r_{\text {ext }}-$ $d=r_{\text {int }} \cos \theta_{2}+r_{\text {ext }} \cos \theta_{1}$. This gives $\sin \left(\theta_{\text {app }}-\pi / 2\right)=$ $\left(r_{\text {int }}-r_{\text {ext }}\right) /\left(r_{\text {int }} \cos \theta_{2}+r_{\text {ext }} \cos \theta_{1}\right)$ or $\cos \theta_{\text {app }}=(1-\epsilon) /$ $\left(\epsilon \cos \theta_{2}+\cos \theta_{1}\right)$, where $\epsilon=r_{\text {int }} / r_{\text {ext }}$. In the limit where $r_{\mathrm{ext}} \sim r_{\text {int }} \ll R, \quad \epsilon=\gamma_{\mathrm{LD}} / \gamma_{\mathrm{LV}}$. Therefore, $\quad \cos \theta_{\text {app }}=$ $\left(\gamma_{\mathrm{LV}}-\gamma_{\mathrm{LD}}\right) /\left(\gamma_{\mathrm{LD}} \cos \theta_{2}+\gamma_{\mathrm{LV}} \cos \theta_{1}\right)$. To satisfy Neumann's triangle, $\gamma_{\mathrm{LD}} \cos \theta_{2}+\gamma_{\mathrm{LV}} \cos \theta_{1}=\gamma_{\mathrm{DV}}$, and we obtain

$$
\cos \theta_{\mathrm{app}}=\frac{\gamma_{\mathrm{LV}}-\gamma_{\mathrm{LD}}}{\gamma_{\mathrm{DV}}}
$$

To verify the validity of Eqs. (B1) and (B2) and, equivalently, Eq. (4) in the main text, we use the data summarized in Table III. While the source data do not include error ranges, it should be noted that the error on contact-angle measurements is typically on the order of $\pm 1^{\circ}$.

\section{APPENDIX C: THE EFFECT OF PINNING A DROPLET OVER A MOVING SURFACE}

It is important to demonstrate that using a capillary tube to pin a droplet above a moving substrate, as done in

TABLE III. Contact angle values.

\begin{tabular}{lcccccccc}
\hline \hline Solid & Droplet & Lubricant & $\gamma_{\mathrm{DV}}$ & $\gamma_{\mathrm{LV}}$ & $\gamma_{\mathrm{LD}}$ & Wrapping & $\cos ^{-1}\left(\gamma_{\mathrm{LV}}-\gamma_{\mathrm{LD}}\right) / \gamma_{\text {eff }}$ & $\theta_{\text {appexp }}$ \\
\hline Epoxy & Water & FC-70 & 72.4 & 17.1 & 56 & No & $122.5^{\circ}$ & $113.1^{\circ}$ \\
Epoxy & $\mathrm{C}_{16} \mathrm{H}_{34}$ & FC-70 & 27.2 & 17.1 & 8.2 & Yes & $69.4^{\circ}$ & $70.5^{\circ}$ \\
Epoxy & $\mathrm{C}_{13} \mathrm{H}_{28}$ & FC-70 & 25.9 & 17.1 & 7.7 & Yes & $67.7^{\circ}$ & $63.5^{\circ}$ \\
Epoxy & $\mathrm{C}_{10} \mathrm{H}_{22}$ & FC-70 & 23.6 & 17.1 & 6.7 & No & $64^{\circ}$ & $60^{\circ}$ \\
Epoxy & $\mathrm{C}_{8} \mathrm{H}_{8}$ & FC-70 & 21.4 & 17.1 & 4.4 & No & $53.6^{\circ}$ & $50.7^{\circ}$ \\
Epoxy & $\mathrm{C}_{6} \mathrm{H}_{14}$ & FC-70 & 18.6 & 17.1 & 2.6 & No & $38.8^{\circ}$ & $40.1^{\circ}$ \\
Epoxy & $\mathrm{C}_{5} \mathrm{H}_{12}$ & FC-70 & 17.2 & 17.1 & 2.5 & No & $31.9^{\circ}$ & $30.8^{\circ}$ \\
Silica & Water $_{\text {Silica }}$ & FC-70 & 72.1 & 17.9 & 52.8 & Yes & $119.6^{\circ}$ & $124.3^{\circ}$ \\
Silica & Ethylene-glycol & FC-70 & 47.3 & 17.9 & 28.2 & Yes & $102.9^{\circ}$ & $89.6^{\circ}$ \\
PMP & Hexadecane & FC-70 & 28 & 17.9 & 7.1 & Yes & $64.4^{\circ}$ & $58.5^{\circ}$ \\
Boehmite & Water & Silicone oil & 72.4 & 20.6 & 41.3 & Yes & $109.5^{\circ}$ & $110^{\circ}$ \\
& Water & Mineral oil & 72.4 & 30 & 48.6 & No & $104.9^{\circ}$ & $105.4^{\circ}$
\end{tabular}

PMP stands for polymethylpentene, and FC-70 is a perfluorinated oil. Data taken from Refs. [6,21].

$\gamma_{\mathrm{eff}}=\gamma_{\mathrm{LV}}+\gamma_{\mathrm{LD}}$ or $\gamma_{\mathrm{DV}}$ with and without a wrapping layer. 


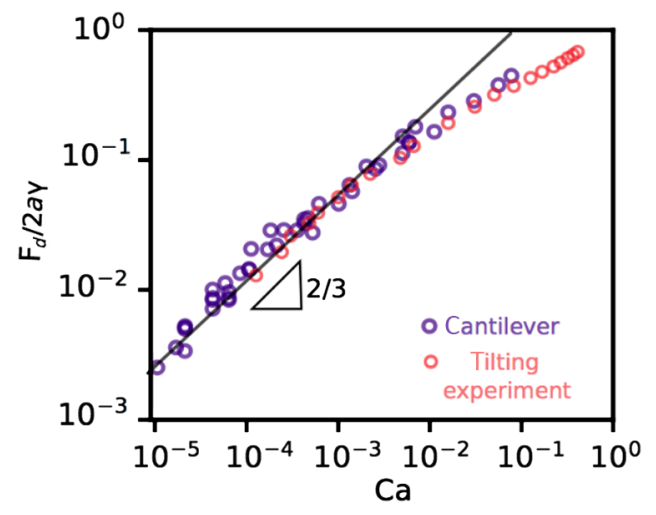

FIG. 9. Comparison between the force measurements performed in our previous paper using a cantilever force sensor (purple circles) [18] and the tilting experiments performed by Keiser et al. (red circles) [31].

our experiment, does not cause a change in the interaction of the droplet with the surface compared to the more conventional case of a droplet moving on a stationary substrate. To prove this, we compare previously reported data from two different experiments which can be used to measure the dissipation force $F_{d}$ acting on a dynamic droplet. Daniel et al. used an experiment similar to that reported in this manuscript: A droplet was pinned above a moving substrate with a cantilever, and the deflection of the cantilever was used to measure $F_{d}$ [18]. Keiser et al. measured the velocity $U$ of droplets at different tilting angles $\theta_{\text {tilt }}=2^{\circ}-90^{\circ}$ on lubricated surfaces (100-cP silicone oil, micropillar surface with high surface fraction $\phi=0.67$ ) [31]. The results from both these experiments can be plotted on the same graph by noting that $F_{d}=W \sin \theta_{\text {tilt }}$, where $W$ is the weight of the droplet. The two results shown in Fig. 9 are in perfect agreement with one another: The droplet experiences the same dissipation force for a moving droplet and a moving substrate. Note, the data in Fig. 13 further demonstrate that there is no difference between the lubricant dynamics behind a pinned droplet and a droplet moving on an inclined surface.

\section{APPENDIX D: MAXIMUM RESOLVABLE ANGLE IN REFLECTION INTERFERENCE CONTRAST MICROSCOPY}

A 2× Mitutoyo Plan Apo infinity-corrected longworking-distance objective with a numerical aperture (NA) of 0.055 is used to generate RICM images. Using the relationship NA $=n \sin \theta$, where $\theta$ is the angle away from normal incidence, we find that $\theta=3.2^{\circ}$. Because the light must pass through first the glass slide $(n=1.54)$ and then the silicone oil $(n=1.4)$, the angle is effectively higher. Using Snell's law, $\sin \theta_{\text {glass }}=1.54 \times \mathrm{NA}$ and $\sin \theta_{\text {lub }}=1.4 \times \mathrm{NA}$, resulting in $\theta_{\text {lub }}=4.4^{\circ}$. Because the objective can collect only light from a cone of illumination defined by $\theta=4.4^{\circ}$, when the angle of the lubricant surface is greater than approximately $4.4^{\circ}$ from the flat surface, reflected light rays are not collected by the objective and the region appears black.

\section{APPENDIX E: EFFECT OF DROPLET VOLUME ON $\boldsymbol{h}_{f}$}

As the size of the droplet decreases, the effect of the droplet radius can no longer be ignored when considering the thickness of the lubricant left behind the moving droplet, and the full form of the scaling equation for $h_{f}$ must be used. Figure 10(a) shows the scaling of $h_{f} / r_{\text {ext }}$ and $h_{f} / r_{\text {ext }}\left(1-r_{\text {ext }} / R\right)^{2 / 3}$ with the capillary number for a $3-\mu l$ droplet. While the two scaling arguments are initially identical, $h_{f} / r_{\text {ext }}$ increases over the course of the experiment while the full scaling term remains much more constant. We can see this effect over a range of droplet
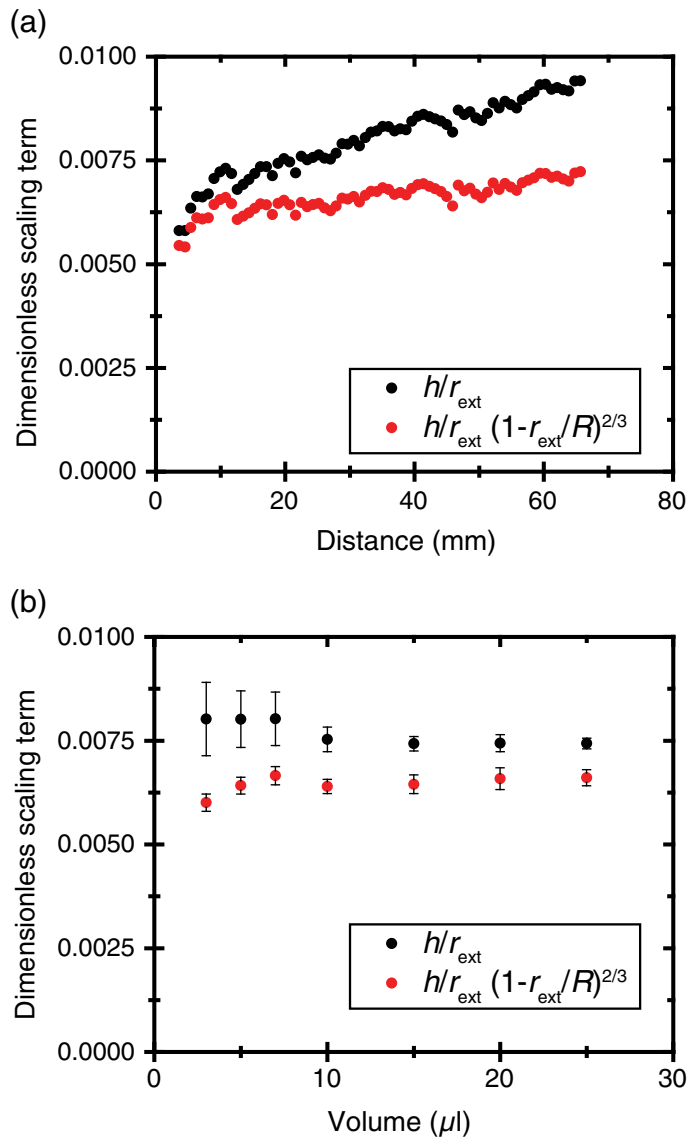

FIG. 10. (a) Comparison of the full and simplified scaling parameter for a $3-\mu \mathrm{l}$ droplet moving at $300 \mu \mathrm{m} / \mathrm{s}$ on a $20-\mathrm{cP}$ silicone-oil-infused surface with $h_{i}=4 \mu \mathrm{m}$, and (b) comparison of the averaged scaling parameter for droplets of different volumes under the same conditions as (a). 
volumes, as shown in Fig. 10(b). While both scaling terms are similar and remain constant for droplets of $10 \mu \mathrm{l}$ or greater in volume, the standard deviation on $h_{f} / r_{\text {ext }}$ is quite high for droplet sizes below approximately $10 \mu \mathrm{l}$, indicating that the value is not constant over the course of the experiment. Conversely, the standard deviation in measurements of the full scaling argument is significantly lower for smaller droplets $(V<10 \mu \mathrm{l})$, although the absolute value of the scaling constant decreases for the smallest droplets. It should be noted that as $r_{\text {ext }}$ approaches $R$, the approximation of the wetting ridge as a torus is no longer valid, and the scaling of $r_{\mathrm{ext}}$ and $h_{f}$ with $C \mathrm{C}^{2 / 3}$ can be expected to deviate from predictions based on a toroidal geometry.

\section{APPENDIX F: EFFECT OF INITIAL CONDITIONS ON $\boldsymbol{h}_{f}$}

We additionally vary the initial lubricant thickness $h_{i}$ for select experimental conditions. While variations in $h_{i}$ change the rate at which the wetting ridge grows, as we discuss in Sec. II C, we see only minor variation in the scaling constant $h_{f} / r_{\text {ext }}$ with $h_{i}$. The Landau-LevichDerjaguin scaling law $h_{f} / r_{\text {ext }} \sim C a_{\mathrm{LV}}^{2 / 3}$ remains unchanged. For 25- $\mu$ l droplets moving at various $C a_{\mathrm{LV}}$ over various silicone oils with $h_{i}=1-4 \mu \mathrm{m}$, the scaling law holds true with a constant prefactor of $\beta=1.55$ (Fig. 11), similar to the value obtained with $h_{i}=4 \mu \mathrm{m}$ for silicone oils and perfluorinated oils over a larger range of capillary numbers $(\beta=1.44)$.

\section{APPENDIX G: WRAPPING LAYER AROUND A STATIC DROPLET}

It is well appreciated in the literature that for a stable wrapping layer of a lubricant over a droplet, the spreading

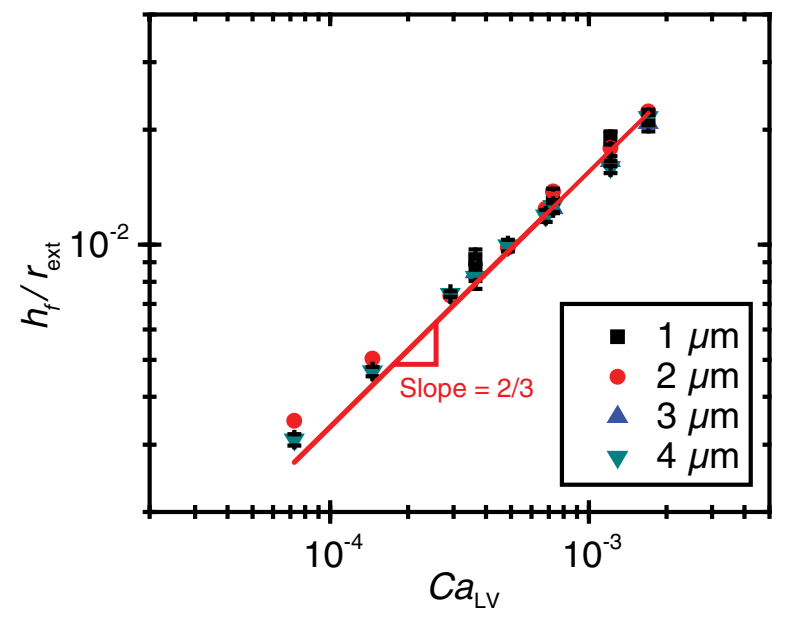

FIG. 11. Scaling of $h_{f} / r_{\text {ext }}$ with $C a_{\mathrm{LV}}$ for various initial thicknesses. Line of best fit in red.

coefficient must be positive, that is, $S_{\mathrm{LD}}=\gamma_{\mathrm{DV}}-\gamma_{\mathrm{LV}}-$ $\gamma_{\mathrm{LD}}>0$, where $\gamma_{\mathrm{DV}}, \gamma_{\mathrm{LV}}$, and $\gamma_{\mathrm{LD}}$ are the interfacial energies of the droplet-vapor, lubricant-vapor, and lubricant-droplet interfaces, respectively [21,22]. As we discuss in the main text, the wetting ridge is a low-pressure region $\left(\Delta P=-\gamma_{\mathrm{LV}} / r_{\mathrm{ext}}\right)$, and lubricant will drain out from the wrapping layer to the wetting ridge until it reaches nanometric thickness, where dispersion van der Waals forces become important.

The nanometric wrapping layer can be stabilized by repulsive van der Waals interactions, that is, if the Hamaker constant $A$ and the resultant disjoining pressure $\Pi(h)=$ $A /\left(6 \pi h^{3}\right)$ in the lubricant film are positive $[47,48]$. If $A<0$, there is attractive van der Waals interactions, and there should not be a stable film. The coefficient $A$ can be estimated by using nonretarded Hamaker constant in Lifshitz theory:

$$
A=\frac{3}{4} K_{B} T\left(\frac{\epsilon_{l}-\epsilon_{d}}{\epsilon_{d}+\epsilon_{l}}\right)\left(\frac{\epsilon_{v}-\epsilon_{l}}{\epsilon_{v}+\epsilon_{l}}\right)+\frac{3 \pi \hbar \nu_{e}}{4 \sqrt{2}} \frac{\left(n_{l}^{2}-n_{d}^{2}\right)\left(n_{v}^{2}-n_{l}^{2}\right)}{\sqrt{\left(n_{d}^{2}+n_{l}^{2}\right)\left(n_{v}^{2}+n_{l}^{2}\right)}\left[\sqrt{\left(n_{d}^{2}+n_{l}^{2}\right)}+\sqrt{\left(n_{v}^{2}+n_{l}^{2}\right)}\right.},
$$

where $\nu_{e} \approx 4 \times 10^{15} \mathrm{~s}^{-1}$ is the plasma frequency of freeelectron gas, while $\epsilon_{d / l / v}$ and $n_{d / l / v}$ are the dielectric constants and refractive indices of the droplet, lubricant, and vapor, respectively $[49,50]$. The equilibrium thickness is then given by a balance between Laplace pressure and the disjoining pressure, i.e., $h_{w, \text { eq }} \sim\left(r_{\text {ext }} A / \gamma\right)^{1 / 3}$, which should be about tens of nanometers.

Using confocal RICM with two illumination lasers ( $\lambda=405 \mathrm{~nm}$ and $\lambda=633 \mathrm{~nm}$ ), it is possible to measure the absolute thickness of $h_{w}$ in the range of $20 \mathrm{~nm}$ to $1.5 \mu \mathrm{m}$. Details of the experimental technique can be found in our previous work [18]. We first track the thickness of a silicone oil wrapping layer $h_{w}(t)$ with time $t$ at the apex of a millimetric sized water droplet. In this case, $A>0$ and we do not expect to see a stable lubricant film.

Yet, under ambient conditions, we find that $h_{w}(t)$ fluctuates rapidly between 50 and $250 \mathrm{~nm}$ as shown in Fig. 12(a). The reason for this seemingly stable film is because of the presence of air draft that entrains the lubricant over the droplet. When the droplet is placed in "still air" by covering it with a transparent container, the wrapping layer is able to drain, and we see dewetting of the lubricant film [Fig. 12(b)]. Strictly speaking, it is not possible to conclusively rule out the presence of lubricant in the dewetted pockets using RICM; however, if there is a lubricant layer in these areas, it is very thin $(<10 \mathrm{~nm})$. 
(a) Silicone oil on water (dynamic)

$S>0, A<0$
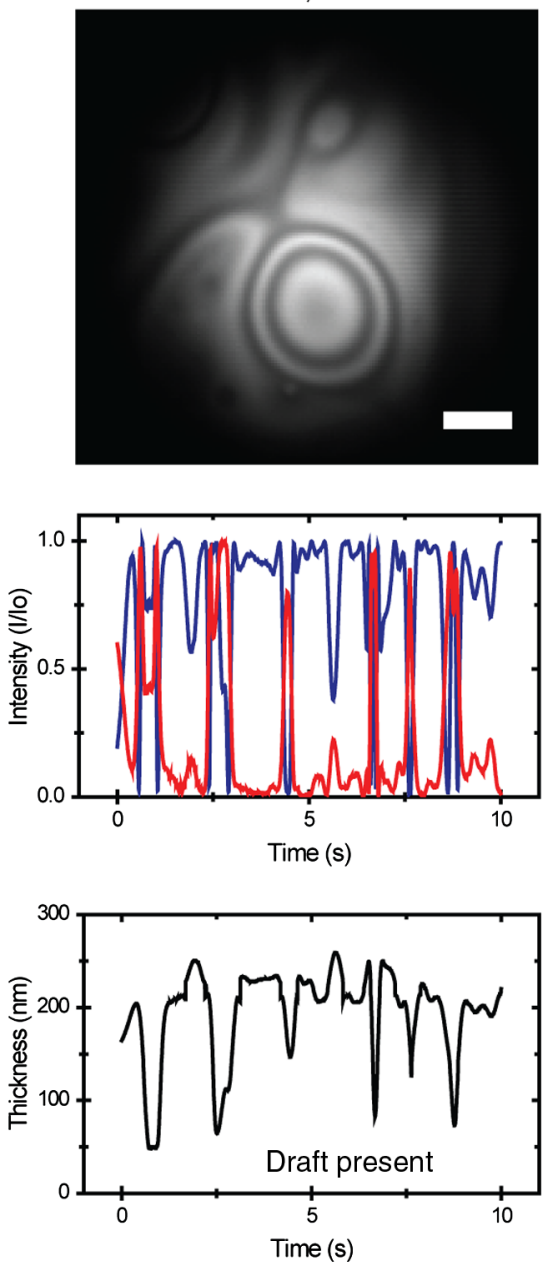

(b)

Silicone oil on water (static)

$S>0, A<0$
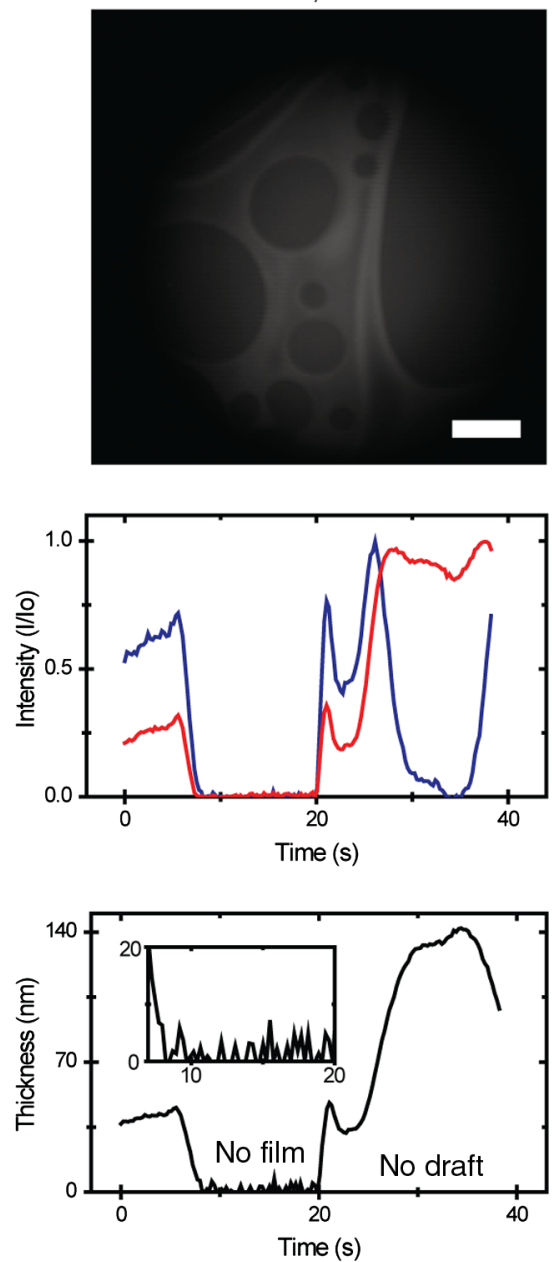

(c)

Silicone oil on glycerine (static)

$S>0, A<0$
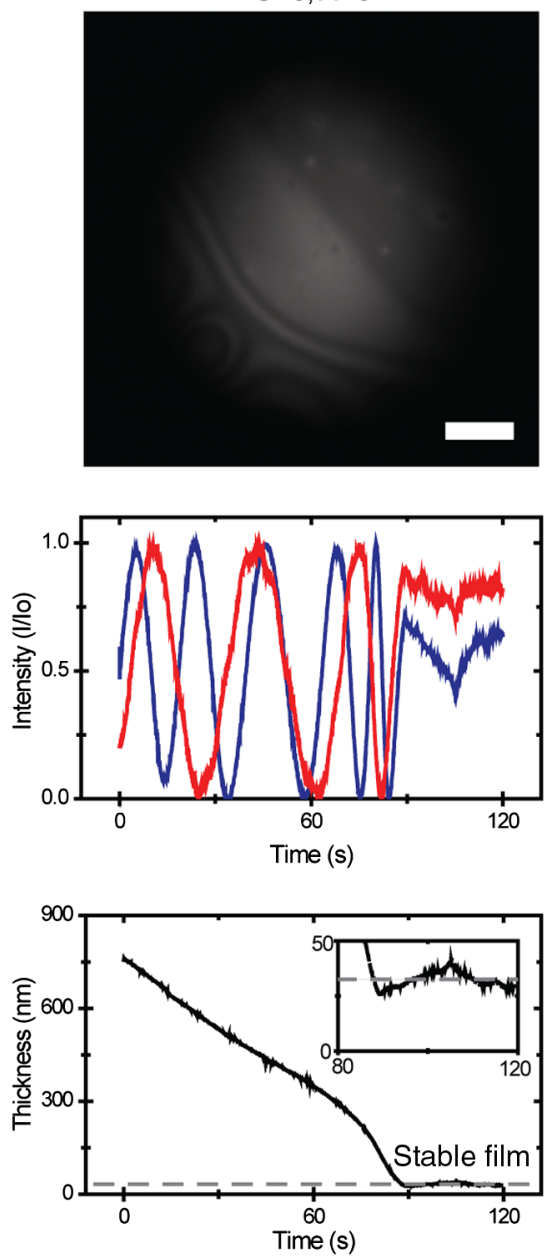

FIG. 12. Evolution of the wrapping layer thickness and profile in time as measured using dual-wavelength RICM. (a) Silicone oil forms thick films on a droplet of water due to the presence of ambient airflow leading to fluctuating thickness measurements. (b) Because of the positive Hamaker constant, thin films of silicone oil on water dewet in the absence of airflow. (c) Silicone oil thin films reach a stable equilibrium thickness on a glycerine droplet due to the negative Hamaker constant, even in the absence of ambient airflow.

We then repeat the same experiment for the wrapping of silicone oil around a droplet of glycerine. In this case, $A>0$ and we expect a stable nanometric film. Initially, we observe a micron-thick lubricant wrapping layer (probably entrained during the initial placement of the droplet), but in still air, the film drains and eventually plateaus to an equilibrium thickness of approximately $40 \mathrm{~nm}$, consistent with the stabilizing effects of a repulsive van der Waals interaction.

\section{APPENDIX H: NUMERICAL FIT OF THE DEPLETION INTEGRAL}

In the main text, we show that the growth of the wetting ridge size $r_{\text {ext }}$ is given by

$$
\ln \left(1-\frac{r_{\mathrm{ext}}}{r_{\infty}}\right)+\frac{r_{\mathrm{ext}}}{r_{\infty}}=-\mu_{1} C a^{4 / 3}\left(\frac{L-\delta}{h_{i}}\right),
$$

where $\quad \mu_{1}=\chi^{2} \beta^{2} /(2 \pi \alpha), \quad \chi=\left\langle h_{f}\right\rangle / h_{f, 0}, \quad$ and $\quad r_{\infty}=$ $h_{i} / \chi \beta C a_{\mathrm{LV}}^{2 / 3}$ is the limiting size of the wetting ridge for a given set of experimental conditions. The integration

TABLE IV. Summary of fitted parameters.

\begin{tabular}{lcrc}
\hline \hline$h_{i}(\mu \mathrm{m})$ & $\alpha$ & $\delta(\mathrm{mm})$ & $\chi$ \\
\hline 4.00 & 0.48 & -0.81 & 0.80 \\
3.06 & 0.45 & 0.30 & 0.80 \\
1.94 & 0.57 & 0.29 & 0.81 \\
1.37 & 0.52 & 0.30 & 0.81 \\
\hline \hline
\end{tabular}


constant $\delta$ is a small positional offset to account for the experimental error in $L$ and the initial wetting ridge size $r_{\text {ext }, 0}$.

To fit the experimental data in Fig. 6(e) in the main text with Eq. (H1) and Eq. (10) in the main text, we use a leastsquares error fitting algorithm in MATLAB with $\alpha, \delta$, and $\chi$ as fitting parameters. All other parameters, such as $h_{i}$ and $\beta$, are experimentally determined. The resulting values are summarized in Table IV.

\section{APPENDIX I: SCALING OF $\boldsymbol{h}_{f}$ OVER AN EXTENDED RANGE Of CAPILLARY NUMBERS}

It is not possible to use the same experimental setup described in the main text to probe $h_{f}$ values at high capillary numbers because the motor cannot reach the required velocity values, and the capillary force from the needle is not sufficient to hold the droplet. Instead, we vary the tilt angles of the surface and use white-light interferometry to measure the thickness of the lubricant film $h_{f}$ immediately following the passage of a droplet. A high-speed camera (Photron SA 1.1) is used to measure the wetting ridge radius $r_{\text {ext }}$ as the droplet passes through the probe. While this experiment cannot give time-resolved data, as is possible with a stationary droplet pinned above a moving surface, testing multiple droplets with wetting ridges of various sizes shows that $h_{f} / r_{\text {ext }}$ is constant for a given capillary number.

Figure 13 is a log-log plot of $h_{f} / r_{\text {ext }}$ vs capillary number for the experiments with inclined surfaces as we describe above (open circles) and with pinned droplets as we describe in the main text (filled circles). We observe that $h_{f} / r_{\mathrm{ext}} \sim \mathrm{Ca}^{2 / 3}$ for $10^{-4}<\mathrm{Ca}<10^{-2}$. This is in good

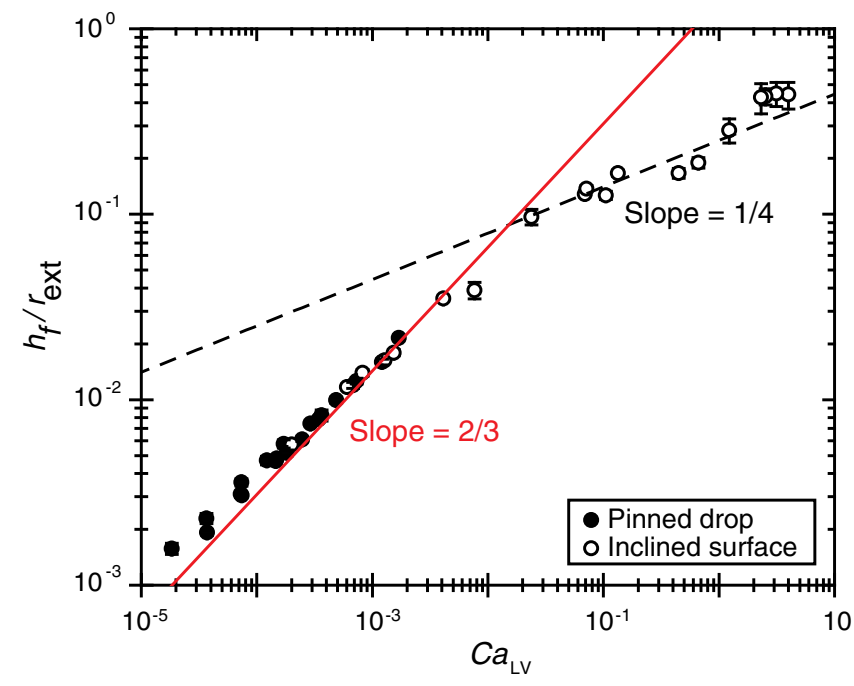

FIG. 13. Plot of $h_{f} / r_{\text {ext }}$ vs capillary number. The red line indicates the line of best fit for $h_{f} / r_{\text {ext }} \sim C a^{2 / 3}$ using pinned droplets. The dashed line shows the approximate slope at high capillary numbers $\left(h_{f} / r_{\text {ext }} \sim C a^{1 / 4}\right)$. agreement with the limits of LLD mechanics observed in the traditional dip-coating literature [31,36-39].

For $C a>10^{-2}$, we observe a change in the scaling behavior, resulting in $h_{f} / r_{\mathrm{ext}} \sim \mathrm{Ca}^{1 / 4}$. Note that the exponent $1 / 4$ is probably an approximation; its exact value (and its derivation) is still an open question and beyond the scope of this study. We further highlight that for $C a<10^{-2}$, the data for inclined surfaces and pinned droplet overlap with each other. This indicates that introducing a needle into the droplet does not affect the droplet geometry and lubricant dynamics significantly.

[1] H. A. Harris, Lubrication in Antiquity, Greece Rome 21, 32 (1974).

[2] A. Fall, B. Weber, M. Pakpour, N. Lenoir, N. Shahidzadeh, J. Fiscina, C. Wagner, and D. Bonn, Sliding Friction on Wet and Dry Sand, Phys. Rev. Lett. 112, 175502 (2014).

[3] O. Reynolds, On the Theory of Lubrication and Its Application to Mr. Beauchamp Tower's Experiments, Including an Experimental Determination of the Viscosity of Olive Oil, Proc. R. Soc. London 40, 191 (1886).

[4] W. H. Briscoe, S. Titmuss, F. Tiberg, R. K. Thomas, D. J. McGillivray, and J. Klein, Boundary Lubrication under Water, Nature (London) 444, 191 (2006).

[5] D. Quéré, Non-Sticking Drops, Rep. Prog. Phys. 68, 2495 (2005).

[6] T.-S. Wong, S. H. Kang, S. K. Y. Tang, E. J. Smythe, B. D. Hatton, A. Grinthal, and J. Aizenberg, Bioinspired SelfRepairing Slippery Surfaces with Pressure-Stable Omniphobicity, Nature (London) 477, 443 (2011).

[7] A. Lafuma and D. Quéré, Slippery Pre-Suffused Surfaces, Europhys. Lett. 96, 56001 (2011).

[8] S. Sunny, G. Cheng, D. Daniel, P. Lo, S. Ochoa, C. Howell, N. Vogel, A. Majid, and J. Aizenberg, Transparent Antifouling Material for Improved Operative Field Visibility in Endoscopy, Proc. Natl. Acad. Sci. U.S.A. 113, 11676 (2016).

[9] D. C. Leslie, A. Waterhouse, J. B. Berthet, T. M. Valentin, A. L. Watters, A. Jain, P. Kim, B. D. Hatton, A. Nedder, K. Donovan et al., A Bioinspired Omniphobic Surface Coating on Medical Devices Prevents Thrombosis and Biofouling, Nat. Biotechnol. 32, 1134 (2014).

[10] P. Kim, T.-S. Wong, J. Alvarenga, M. J. Kreder, W. E. Adorno-Martinez, and J. Aizenberg, Liquid-Infused Nanostructured Surfaces with Extreme Anti-Ice and Anti-Frost Performance, ACS Nano 6, 6569 (2012).

[11] M. J. Kreder, J. Alvarenga, P. Kim, and J. Aizenberg, Design of Anti-Icing Surfaces: Smooth, Textured or Slippery?, Nat. Rev. Mater. 1, 15003 (2016).

[12] G. Mistura and M. Pierno, Drop Mobility on Chemically Heterogeneous and Lubricant-Impregnated Surfaces, Adv. Phys. X 2, 591 (2017).

[13] J. S. Wexler, I. Jacobi, and H. A. Stone, Shear-Driven Failure of Liquid-Infused Surfaces, Phys. Rev. Lett. 114, 168301 (2015).

[14] K. Rykaczewski, S. Anand, S. B. Subramanyam, and K. K. Varanasi, Mechanism of Frost Formation on LubricantImpregnated Surfaces, Langmuir 29, 5230 (2013). 
[15] P. Kim, M. J. Kreder, J. Alvarenga, and J. Aizenberg, Hierarchical or not? Effect of the Length Scale and Hierarchy of the Surface Roughness on Omniphobicity of Lubricant-Infused Substrates, Nano Lett. 13, 1793 (2013).

[16] J.-H. Kim and J. P. Rothstein, Delayed Lubricant Depletion on Liquid-Infused Randomly Rough Surfaces, Exp. Fluids 57, 81 (2016).

[17] J. S. Wexler, A. Grosskopf, M. Chow, Y. Fan, I. Jacobi, and H. A. Stone, Robust Liquid-Infused Surfaces through Patterned Wettability, Soft Matter 11, 5023 (2015).

[18] D. Daniel, J. V. I. Timonen, R. Li, S. J. Velling, and J. Aizenberg, Oleoplaning Droplets on Lubricated Surfaces, Nat. Phys. 13, 1020 (2017).

[19] C. Semprebon, G. McHale, and H. Kusumaatmaja, Apparent Contact Angle and Contact Angle Hysteresis on Liquid Infused Surfaces, Soft Matter 13, 101 (2017).

[20] M. Tress, S. Karpitschka, P. Papadopoulos, J. H. Snoeijer, D. Vollmer, and H.-J. Butt, Shape of a Sessile Drop on a Flat Surface Covered with a Liquid Film, Soft Matter 13, 3760 (2017).

[21] F. Schellenberger, J. Xie, N. Encinas, A. Hardy, M. Klapper, P. Papadopoulos, H.-J. Butt, and D. Vollmer, Direct Observation of Drops on Slippery Lubricant-Infused Surfaces, Soft Matter 11, 7617 (2015).

[22] J. D. Smith, R. Dhiman, S. Anand, E. Reza-Garduno, R. E. Cohen, G. H. McKinley, and K. K. Varanasi, Droplet Mobility on Lubricant-Impregnated Surfaces, Soft Matter 9, 1772 (2013).

[23] J. H. Guan, G. G. Wells, B. Xu, G. McHale, D. Wood, J. Martin, and S. Stuart-Cole, Evaporation of Sessile Droplets on Slippery Liquid-Infused Porous Surfaces (Slips), Langmuir 31, 11781 (2015).

[24] T. Young, An Essay on the Cohesion of Fluids, Phil. Trans. R. Soc. London 95, 65 (1805).

[25] I. Cantat, S. Cohen-Addad, F. Elias, F. Graner, R. Höhler, O. Pitois, F. Rouyer, and A. Saint-Jalmes, Foams: Structure and Dynamics (Oxford University Press, Oxford, 2013).

[26] F. C. Goodrich, The Mathematical Theory of Capillarity. I, Proc. R. Soc. A 260, 481 (1961).

[27] See Supplemental Material at http://link.aps.org/ supplemental/10.1103/PhysRevX.8.031053 for interferometric time-lapse videos of the lubricant dynamics underneath, surrounding and wrapping around droplets moving on SLIPS.

[28] J. de Ruiter, F. Mugele, and D. van den Ende, Air Cushioning in Droplet Impact. I. Dynamics of Thin Films Studied by Dual Wavelength Reflection Interference Microscopy, Phys. Fluids 27, 012104 (2015).

[29] B. Derjaguin, Thickness of Liquid Layer Adhering to Walls of Vessels on their Emptying and the Theory of Photo- and Motion-Picture Film Coating, C. R. (Dokl.) Acad. Sci. URSS 39, 13 (1943).

[30] L. Landau and V. Levich, Dragging of a Liquid by a Moving Plate, Acta Physicochim. URSS 17, 42 (1942).

[31] A. Keiser, L. Keiser, C. Clanet, and D. Quéré, Drop Friction on Liquid-Infused Materials, Soft Matter 13, 6981 (2017).

[32] R. F. Probstein, Physicochemical Hydrodynamics: An Introduction (John Wiley \& Sons, New York, 2005).
[33] H. Lhuissier, Y. Tagawa, T. Tran, and C. Sun, Levitation of a Drop over a Moving Surface, J. Fluid Mech. 733, R4 (2013).

[34] R. C. A. van der Veen, T. Tran, D. Lohse, and C. Sun, Direct Measurements of Air Layer Profiles under Impacting Droplets Using High-Speed Color Interferometry, Phys. Rev. E 85, 026315 (2012).

[35] K.-C. Park, P. Kim, A. Grinthal, N. He, D. Fox, J. C. Weaver, and J. Aizenberg, Condensation on Slippery Asymmetric Bumps, Nature (London) 531, 78 (2016).

[36] R. P. Spiers, C. V. Subbaraman, and W. L. Wilkinson, Free Coating of a Newtonian Liquid onto a Vertical Surface, Chem. Eng. Sci. 29, 389 (1974).

[37] F. P. Bretherton, The Motion of Long Bubbles in Tubes, J. Fluid Mech. 10, 166 (1961).

[38] D. A. White and J. A. Tallmadge, Theory of Drag Out of Liquids on Flat Plates, Chem. Eng. Sci. 20, 33 (1965).

[39] M. Maleki, M. Reyssat, F. Restagno, D. Quéré, and C. Clanet, Landau-Levich Menisci, J. Colloid Interface Sci. 354, 359 (2011).

[40] J. H. Snoeijer, G. Delon, M. Fermigier, and B. Andreotti, Avoided Critical Behavior in Dynamically Forced Wetting, Phys. Rev. Lett. 96, 174504 (2006).

[41] J. H. Snoeijer and B. Andreotti, Moving Contact Lines: Scales, Regimes, and Dynamical Transitions, Annu. Rev. Fluid Mech. 45, 269 (2013).

[42] C. Urata, G. J. Dunderdale, M. W. England, and A. Hozumi, Self-Lubricating Organogels (Slugs) with Exceptional Syneresis-Induced Anti-Sticking Properties against Viscous Emulsions and Ices, J. Mater. Chem. 3, 12626 (2015).

[43] J. Cui, D. Daniel, A. Grinthal, K. Lin, and J. Aizenberg, Dynamic Polymer Systems with Self-Regulated Secretion for the Control of Surface Properties and Material Healing, Nat. Mater. 14, 790 (2015).

[44] J. Yuan, X. Liu, O. Akbulut, J. Hu, S. L. Suib, J. Kong, and F. Stellacci, Superwetting Nanowire Membranes for Selective Absorption, Nat. Nanotechnol. 3, 332 (2008).

[45] B. Douglas, Tracker Video Analysis and Modeling Tool, http://physlets.org/tracker/ (July 30, 2017).

[46] T. H. Besseling, J. Jose, and A. Blaaderen, Methods to Calibrate and Scale Axial Distances in Confocal Microscopy as a Function of Refractive Index, J. Microsc. 257, 142 (2015).

[47] P.-G. de Gennes, F. Brochard-Wyart, and D. Quéré, Capillarity and Wetting Phenomena: Drops, Bubbles, Pearls, Waves (Springer Science \& Business Media, New York, 2013).

[48] F. Brochard-Wyart, J. M. Di Meglio, D. Quére, and P.-G. de Gennes, Spreading of Nonvolatile Liquids in a Continuum Picture, Langmuir 7, 335 (1991).

[49] J. N. Israelachvili, Intermolecular and Surface Forces, revised 3rd ed. (Academic Press, New York, 2011), pp. 253-271.

[50] V. A. Parsegian, van der Waals Forces: A Handbook for Biologists, Chemists, Engineers, and Physicists (Cambridge University Press, Cambridge, England, 2005). 